\title{
A Semiempirical Methodology to Characterise a Family of Microvibration Sources
}

\author{
S. De Lellis ${ }^{\mathrm{a}, *}$, A. Stabile ${ }^{\mathrm{a}}$, G. S. Aglietti ${ }^{\mathrm{a}}$, G. Richardson ${ }^{\mathrm{b}}$ \\ ${ }^{a}$ Surrey Space Centre, University of Surrey, Guildford, GU2 7XH, UK \\ ${ }^{b}$ Surrey Satellite Technology Limited (SSTL), Guildford, GU2 7YE, UK
}

\begin{abstract}
It is well documented that reaction wheels are among the most significant microvibration sources in space applications. These components, despite being nominally identical, can show differences in the generated signals due to manufacturing imperfections in their internal elements, such as ball bearing, internal and external race. In this article a methodology to account for those variations in microvibration predictions is proposed, aiming at generating a disturbance input matrix that encompasses the effects of a family of reaction wheels. With such a tool, it is possible to provide a more accurate microvibration budget at an early stage of the mission, reducing the uncertainty margin usually applied to quantify reaction wheel effects on the structure. As a consequence better designs are produced faster and cheaper. This allows for more flexibility in the mission design and reduces the degree of uncertainties in the predictions. Furthermore, it is shown that the proposed approach is able to characterise the effects of the entire family of wheels by considering only a limited number. The methodology is validated by assessing the microvibration excitation on different structures, including a real space structure with various reaction wheel mounting configurations.

Keywords: Reaction wheel; Cross-Correlation; Power Spectral Density; Mechanical defect
\end{abstract}

*Corresponding author. E-mail: s.delellis@surrey.ac.uk 


\section{Introduction}

In the last years the need of satellites with increased resolution has led to optical systems with large area for image capturing. Due to the harsh constraints in terms of available volume and mass from the launcher companies, such systems have to be lightweight, [1], often resulting in flexible structure susceptible to vibrations that need to be minimised. In particular, optical payloads can be strongly affected by disturbances generated by actuators and mechanisms causing imaging quality degradation, [2], especially at high frequency. This category of disturbances defines microvibrations. The majority of institutional science mission are sensitive to microvibration as are all high resolution optical missions with an optical terminal. Managing microvibration on these missions add significant cost and schedule.

There are a large number of vibration sources on board the spacecraft. Examples are propulsion thrusters, whose main disturbances occur during opening and closing of the mechanical valve on the nozzle, [3, 4], or antenna pointing mechanisms, which are used to point antennas and mobile mirrors. Different studies, such as $[5,6]$, show the importance of these vibrations on the Space Interferometry Mission and the relevance of the disturbances coming from microvibration sources.

Cryocoolers represent another example of vibration sources. They are used to keep parts of the spacecraft within the desired temperature. [7] shows the disturbances generated by these components on the James Webb Space Telescope and their effect on the mission design. Last but not least, Reaction Wheels (RW) have been extensively studied and analysed. These components are used as control actuators and they were found to be influential in several space missions. An example is the Advanced X-Ray Astrophysics Facility where an isolation system, using viscous material, was required to damp out the generated vibrations, [8]. Examples of Earth observation mission are also available, such as [9] where microvibrations generated on board SSTL 300 S1 platform are studied and an isolation system is discussed to attenuate microvibrations.

When it comes to the evaluation of the structural behaviour of the spacecraft, uncertainties can arise, [10]. These can be structure related, [11-14] or source related, [15]. The latter are the focus of this paper. They mainly include imperfections due to manufacturing processes in nominally identical source equipment that can lead to different behaviour of the structure in terms of generated vibration response, $[16,17]$. This article focuses on 
the microvibrations generated by reaction wheels and particularly the variation in microvibration between wheels which should be the same. Test data shows that there is considerable variation of the noise generated by reaction wheel which belong to the same product family. Ideally, all noise sources on a spacecraft should be measured individually and each spacecraft should be assessed based on the measurement of the noise sources it uses. This is not feasible mainly for time constraints. In addition during the design process, the specific source component to be placed on the spacecraft is selected from the batch at a very late stage of the mission design. Hence, in order to account for these variations in the analysis, a safety factor is usually employed, [18-20]. In the design of a space mission, there are a large number of components and subsystems, hence the safety factor for the whole system becomes a complex issue,[21].

Generally speaking, the higher is the degree of uncertainty associated to a subsystem, the higher is the safety margin associated to it which corresponds to higher redundancy, mass and cost, [22]. As such, a reduction of this parameter would be beneficial if the system is robust enough to withstand it. Hence, this paper aims at reducing the uncertainty margin allocated for microvibration sources, in particular reaction wheels, by proposing an innovative method for more accurate vibration predictions applicable at an early stage of the mission design.

In this framework, the safety factor associated to disturbances produced by RWs plays an important role; literature shows that vibrations produced by these devices are among the most significant on board the spacecraft, [23, 24]. This justifies the great amount of research on RWs, in terms of mathematical models, [25, 26], experimental validation and characterisation, [27, 28].

Disturbances in the RWs are mainly due to imperfections inside the wheel components coming from manufacturing tolerances, as extensively described in $[29-31]$.

The identification of these manufacturing defects is crucial as they can couple with the structural modes of the wheel producing an amplification of the reaction wheel signature. The common procedure is the estimation of these couplings and the evaluation of the effects of the RW on the spacecraft at these specific frequencies. In order to detect them, an equivalent sine load is determined at each coupling frequency from the produced disturbance of the reaction wheel; then, a frequency response analysis is run on the satellite under test at that frequency. The drawback of this procedure stays in 
the limited number of frequency cases considered. Conversely, it is shown in the literature that not only the coupling between hamonics and RW modes can amplify the RW signature, but also the interaction between vibrations generated by defects in the sources (such as reaction wheels) and spacecraft elements can be significant, $[32,33]$. Hence, a more integrated approach should be adopted. This can be obtained by defining the spectral power matrix of the wheel: the spectral power of each of the six disturbances at every frequency step is computed. This is a fairly common procedure in the literature, as reported in $[34,35]$. The computed matrix, i.e. RW disturbance input matrix, is then applied to the structure and responses are computed. Despite providing a more thorough approach than the previous procedure, the disturbance matrix is usually assumed to be diagonal, [34]. This is justified by the uncorrelated behaviour between signals along different directions (corresponding to the off-diagonal terms of the disturbance matrix) and their small magnitude compared to the diagonal ones.

This article shows that this approximation only holds in some specific circumstances, extending what has already been achieved in [15, 35].

A methodology is proposed to provide better estimations of RW disturbances on the structure, allowing the evaluation of a microvibration budget at an early stage of the mission design for a family of RWs. Indeed, from the measurement of one reaction wheel a full disturbance matrix can be generated. However if measurements are available from multiple reaction wheels it is not obvious how to generate one generalised disturbance matrix that represents either the typical behaviour or the worst case.

Hence, the suggested methodology allows first, to assess the effects of a family of RWs vibrations on the spacecraft without selecting the specific wheel. Second, to reduce the safety margins required in the response predictions compared to the available methodologies. Third, to obtain a more accurate and thorough understanding of the nature of the mathematical terms describing RW imperfections by quantifying the response variation in different structures of the disturbance input matrix. Lastly, it is able to characterise the typical behaviour of a family of microvibration sources by considering only a limited number of devices. As extensively explained in this paper, the main element of novelty of this study lies in the implemented approach to retrieve the generalised disturbance input matrix and in its applicability to a generic structure. 


\section{Theory}

\subsection{Power Spectral Density in Vibration Analysis}

As already highlighted, RWs can be characterised through spectral analysis via the definition of a Power Spectral Density matrix $\Psi_{\mathbf{I N}}(f)$. This is made of diagonal terms $\Psi_{i i}(f)$ representing the Fourier transform of the autocorrelation function $C_{x x}(t)$ of the signal $x(t),[36]$, and they are called Power Spectral Density (PSD) terms. These quantities are real and positive. Similarly, the off-diagonal terms of the matrix are the Cross Power Spectral Density (CPSD) and are defined as the Fourier transform of the cross-correlation function $C_{x y}(t)$ between signals $x(t)$ and $y(t)$. CPSD are complex values. Hence, when a RW is defined in the frequency domain a 6x6 matrix containing PSD on the diagonal and CPSD on the off-diagonal positions is generated, such that:

$$
\left[\begin{array}{cccc}
P S D_{x x}(f) & \ldots & \ldots & C P S D_{x M z}(f) \\
\ldots & \ldots & \ldots & \ldots \\
\ldots & \ldots & \ldots & \ldots \\
C P S D_{x M z}^{*}(f) & \ldots & \ldots & P S D_{M z M z}(f)
\end{array}\right]
$$

where the apex ${ }^{*}$ indicates the complex conjugate of the term. The common approach neglects the off-diagonal terms as the matrix is thought to be diagonally dominant, [34]. The assumption is usually driven by the fact that phases between signals along different directions are random and uncorrelated and the magnitude of these terms is negligible compared to the diagonal ones. This is true in some cases but generally, when dealing with RW mechanical defects, there can be a relation between signals in different directions. This happens as the defects represent a physical phenomenon inside the wheel, thus a correlation may exist. Such scenario can be seen in Figure 1 where a defect is shown on the ball and the recorded disturbance in time domain. An impulse train is generated due to the defect, whose features depend on the properties of the mechanical components involved (diameter of the balls, surface roughness, material, ...), [17]. [35, 37] are two relevant studies on this topic as they highlight the importance of the CPSD terms in the definition of the disturbance input matrix of RWs. Both articles show the modelling, testing and validation for optical performance prediction of Space Interferometry Mission. Results clearly show that including CPSD in the disturbance matrix of RWs alters the predictions. Hence, the authors 


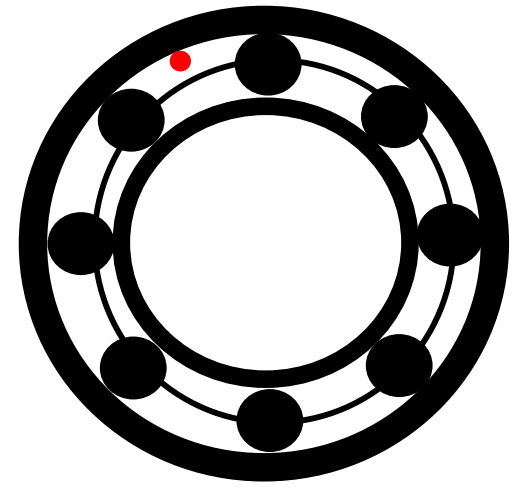

(a) Ball bearing defects

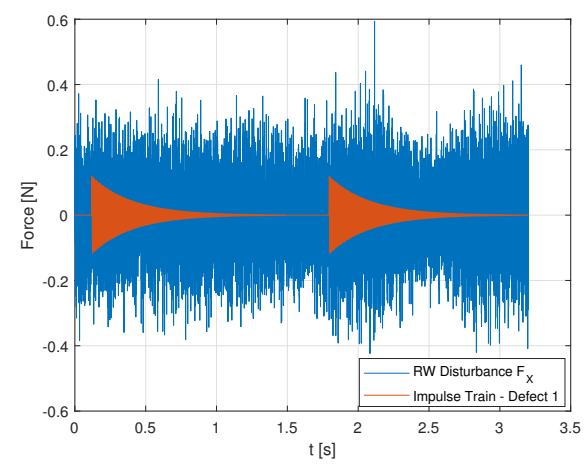

(b) Time domain disturbance

Figure 1: Train impulse due to defect

recommend to fully populate the matrix for future applications. This paper, among its objectives, aims at enhancing the work described in [35] with a more detailed and thorough understanding of the effects and the nature of CPSD terms on the response. The following paragraph shows an example of the relevance of CPSDs in vibration predictions.

\subsection{Practical Example}

A square panel is considered: it is modelled using shell elements and its main properties are reported in Table 1.

The panel is subjected to two load cases, i.e. Directional $(D I R)$ and Ro-

\begin{tabular}{cc}
\hline Property & Value \\
\hline Dimensions (X x Y x Z) $[\mathrm{m}]$ & $1 \times 1 \times 0.01$ \\
Young modulus $[\mathrm{GPa}]$ & 69.9 \\
Density $\left[\mathrm{kg} / \mathrm{m}^{3}\right]$ & 2810 \\
Poisson ratio [ ] & 0.33 \\
Boundary conditions & Blocked at the 4 nodes \\
\hline
\end{tabular}

Table 1: Structural panel properties

tational $(R O T)$. These are described in Figure 2, where the panel is shown with four blocked nodes in the two load cases. It can be seen that the excitation $\vec{F}$ is applied at the centre of the panel with an offset with respect to the plane XY represented by a rigid connection. The two load cases are described 


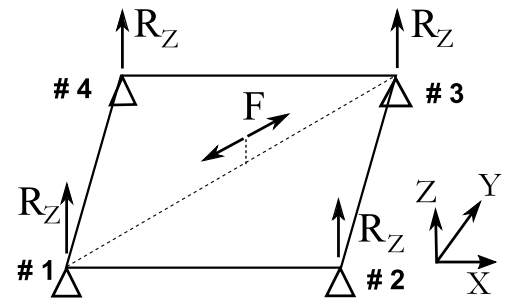

(a) Panel - Oblique Case

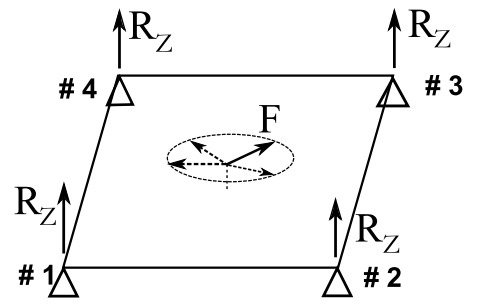

(b) Panel - Rotating Case

Figure 2: Directional and Rotational Case

using sinusoidal loads. Such force decomposition is helpful to show that, despite having the same diagonal elements in the two cases for the disturbance input matrix $\boldsymbol{\Psi}_{\mathbf{I N}}$, as shown in Equation 2, different physical problems are described. This difference is expressed through the off-diagonal terms of the matrix. The first case is a force acting along the diagonal whose magnitude varies sinusoidally, Figure 2a. The second case is a force of fixed amplitude which rotates on a plane parallel to the panel, Figure $2 \mathrm{~b}$. In particular, by considering the force $\vec{F}$ in the $D I R$ case to be expressed as $F_{X}(t)=C \cdot \sin (\omega t)$ and $F_{Y}(t)=D \cdot \sin (\omega t)$, a diagonal force with $C=D$ can be taken as an example to clearly show the effect of the phase shift. In fact, when adding a phase shift of $\pi / 2$ the force shows a rotating behaviour (which is what happens in the $R O T$ case $F_{X}(t)=C \cdot \sin (\omega t)$ and $\left.F_{Y}(t)=D \cdot \cos (\omega t)\right)$. However, it can be noted that the same outcome could be obtained by considering $C \neq D$. In such scenario, the $D I R$ case would still be directional but act on a different direction compared to the case $C=D$, where the force is diagonal. Analogously, in the ROT case the described pattern would be elliptical rather than circular. The terms of $\boldsymbol{\Psi}_{\text {IN }}$ can be expressed as a function of the Fourier transform of the signal itself using the Wiener-Khinchin theorem, [38].

$$
\begin{aligned}
& \Psi_{I N-11}(f)=\left|F F T\left(F_{X}\right)\right|^{2}=\Psi_{I N-22}(f) \\
& \Psi_{I N-12}(f)=F F T\left(F_{X}(t)\right) \cdot F F T\left(F_{Y}(t)\right)=\Psi_{I N-21}^{*}(f)
\end{aligned}
$$

The aim of this example is the evaluation of the off-diagonal terms influence through the reaction force $R_{Z}$ using the following Equation:

$$
\boldsymbol{\Psi}_{\text {OUT }}(f)=\mathbf{H}(f) \cdot \boldsymbol{\Psi}_{\mathbf{I N}}(f) \cdot \mathbf{H}^{H}(f)
$$

where the apex $\mathrm{H}$ indicates the Hermitian of the matrix. $\Psi_{\text {OUT }}$ contains information about the constraint force $R_{Z}$ at the four nodes. First, the 
transfer function matrix $\mathbf{H}$ of the panel is built. This is a $4 \times 2$ matrix with $H_{i j}$ representing the reaction force $R_{Z}$ at node $i$ due to a force applied along direction $j$ (being $j=1,2$ respectively directions $X$ and $Y$ ). Secondly, the disturbance input matrix $\boldsymbol{\Psi}_{\text {IN }}$ is reported in Eq. 4. It can be noticed that this matrix has the same diagonal in both cases DIR and $R O T$. This is because the square of the Fourier transform loses the phase information, see Eq. 2, hence there is no difference between the sine and cosine components.

$$
\boldsymbol{\Psi}_{\mathbf{I N}}(f)=\left[\begin{array}{ll}
\Psi_{I N-11}(f) & \Psi_{I N-12}(f) \\
\Psi_{I N-12}^{*}(f) & \Psi_{I N-22}(f)
\end{array}\right]
$$

The only difference between $\boldsymbol{\Psi}_{\mathbf{I N}}$ in the two cases stays in the CPSD terms. Thus studying the DIR and ROT cases in the frequency domain implies looking at the effects of the off diagonal terms in the input matrix. Table 2

\begin{tabular}{ccc}
\hline Case & $\begin{array}{c}\mathbf{R}_{\mathbf{Z}} \text { at node } 1 \\
{[\mathrm{~N}]}\end{array}$ & $\begin{array}{c}\mathbf{R}_{\mathbf{Z}} \text { at node } 2 \\
{[\mathrm{~N}]}\end{array}$ \\
\hline$R O T \mathrm{w} \Psi_{i j}$ & 0.0016 & 0.0016 \\
$R O T \mathrm{w} / \mathrm{o} \Psi_{i j}$ & 0.0016 & 0.0016 \\
$D I R \mathrm{w} \Psi_{i j}$ & 0.0022 & 0 \\
$D I R \mathrm{w} / \mathrm{o} \Psi_{i j}$ & 0.0016 & 0.0016 \\
\hline
\end{tabular}

Table 2: Rms values for the panel frequency simulations

summarises the results obtained from this simulation at node 1 and 2 using Eq. 3. It can be clearly noticed that no difference is shown between $R O T \mathrm{w} /$ $\Psi_{i j}$ and $R O T w / o \Psi_{i j}\left(\Psi_{i j}=0\right)$ cases when the same node is considered, thus $\Psi_{i j}$ terms do not affect the simulations. In addition to that, the RMS value for both ROT cases is the same at the two different nodes. This is expected as they experiment the same value of $R_{Z}$ being the input an in plane rotational force. Conversely, in the DIR cases Table 2 shows discrepancies between $D I R$ $w / \Psi_{i j}$ and $D I R w / o \Psi_{i j}$. DIR $w / \Psi_{i j}$ case, indeed, results in a null RMS value for $R_{Z}$ at node 2 , which is in line with what is expected, whereas it is $0.0022 N$ at node 1 . The importance of $\Psi_{i j}$ terms is finally clear looking at case $D I R w / o \Psi_{i j}$. Here the input matrix $\Psi_{\mathbf{I N}}$ is built setting $\Psi_{i j}$ to zero and this generates physically wrong results, as the case provides the same results as ROT, which represents a different physical case. This example 
shows that $\Psi_{i j}$ terms in the input matrix contain the phase information and they are influential when a directional excitation is considered (DIR cases). Conversely, in the case of a rotational force (ROT cases) no difference is shown. It is important to point out that the phase content of the $\Psi_{i j}$ terms is not intended as spatial phase. Instead, the phase shift contained in the $\Psi_{i j}$ terms refers to the time delay between signal $X$ and $Y$, hence this information depends on the signal considered, rather than the physical directions. From this example it can be highlighted that $\Psi_{i j}$ terms can affect the description of the physical scenario described by the input matrix $\Psi_{\mathbf{n}}$.

\section{3. $R W$ Harmonics}

Similarly to the example just described, RWs generated disturbances can be represented as rotational or non-rotational signals. These are due to geometrical defects in the mechanical components of the wheel occurring at specific frequencies $f_{i}$ which can be normalised with respect to the rotational speed of the RW $\omega$ to obtain the harmonics, $[39,40]$. Their definition is given as follows:

$$
h=\frac{f}{\omega}
$$

here, $f$ is the frequency where the defect occurs and $\omega$ is the rotational speed of the wheel, expressed in Hz. Different defects can be pinpointed and for each of them the frequency content changes depending on the speed of the RW $\omega$, see Figure 3. In order to locate the harmonics generated by these defects in the frequency domain, different techniques are available. Here a semiempirical procedure is adopted which can reveal the harmonics in a full range of velocities of RW by data processing through a MATLAB tool. The main peaks are identified at each rotational speed of the wheel and they are then compared. Those peaks showing consistency all over the speed range are very likely to be harmonics. The procedure is iterated for radial and axial forces as well as radial torques. This method is borrowed from [41], developed further, and used in this paper for the identification of harmonics. Its description is provided in the following pages.

\subsection{RW Harmonics Identification}

From this point on, every section of the article will recall the flow chart in Figure 4 which will be used as a guideline for the description of the new methodology.

The model used in this study for harmonics identification is based on [41]. 


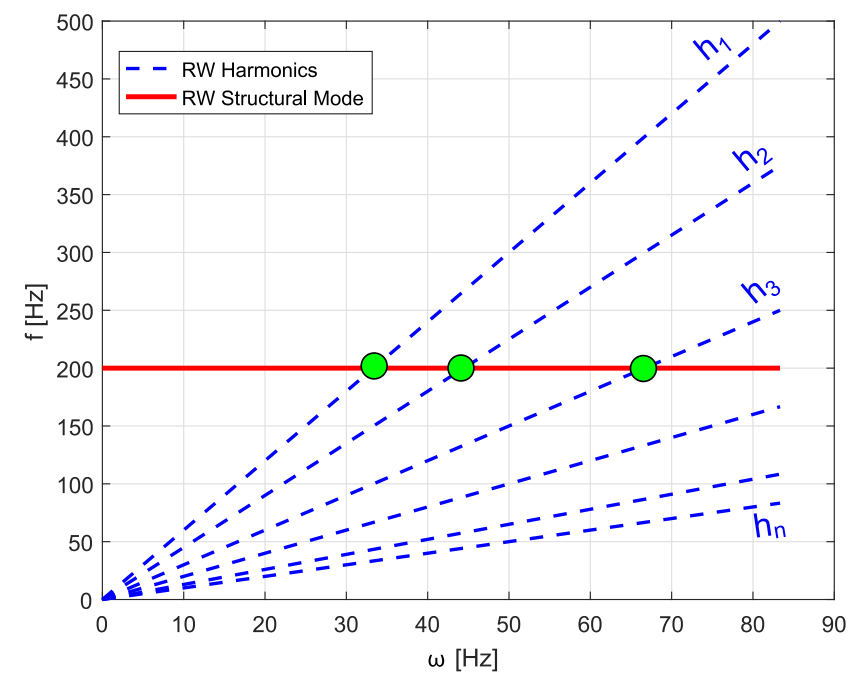

Figure 3: Typical behaviour for RW harmonics (dashed lines) and RW structural modes (solid line). Circles identify the crossing between RW structural modes and harmonics.

The approach used to identify RW harmonics relies on the data collected at different RW rotational speeds $\omega$. In particular, radial force harmonics of the wheel are found looking at the vibrations produced along $F_{X}$ and $F_{Y}$,axial harmonics looking at $F_{Z}$, radial torque harmonics looking at $M_{X}, M_{Y}$ and axial $M_{Z}$. Consider the radial force harmonics. The procedure is shown in Figure 5. First, the power spectral density $\Psi_{X X}(f)$ from time signal $F_{X}(t)$ is evaluated. This is performed using the Welch method, [42], also known as modified periodogram method. In order to find the meaningful peaks representing the harmonics, a threshold is introduced as defined in Eq. 6, and all the frequency steps whose spectral amplitude is above this value are considered as possible harmonics locations. The procedure is iterated at all the wheel speeds $\underline{\omega}$ available in the measurements.

$$
D T(\omega)=\mu_{\text {noise }}(\omega)+N_{\sigma} \cdot \sigma_{\text {noise }}(\omega)
$$

$\mu_{\text {noise }}$ and $\sigma_{\text {noise }}$ represent the mean and standard deviation of the spike amplitudes and they are extrapolated from the histogram of the considered $\Psi$ amplitude (for a full explanation refer to [41]). $N_{\sigma}$ is a user defined value and it depends on the signal-to-noise ratio of the data. $\mu_{\text {noise }}$ and $\sigma_{\text {noise }}$ change as the wheel speed varies. In the case under analysis RW data were acquired at a fairly high sampling frequency, i.e. $2560 \mathrm{~Hz}$, which allows $N_{\sigma}$ to be 2.5 , 


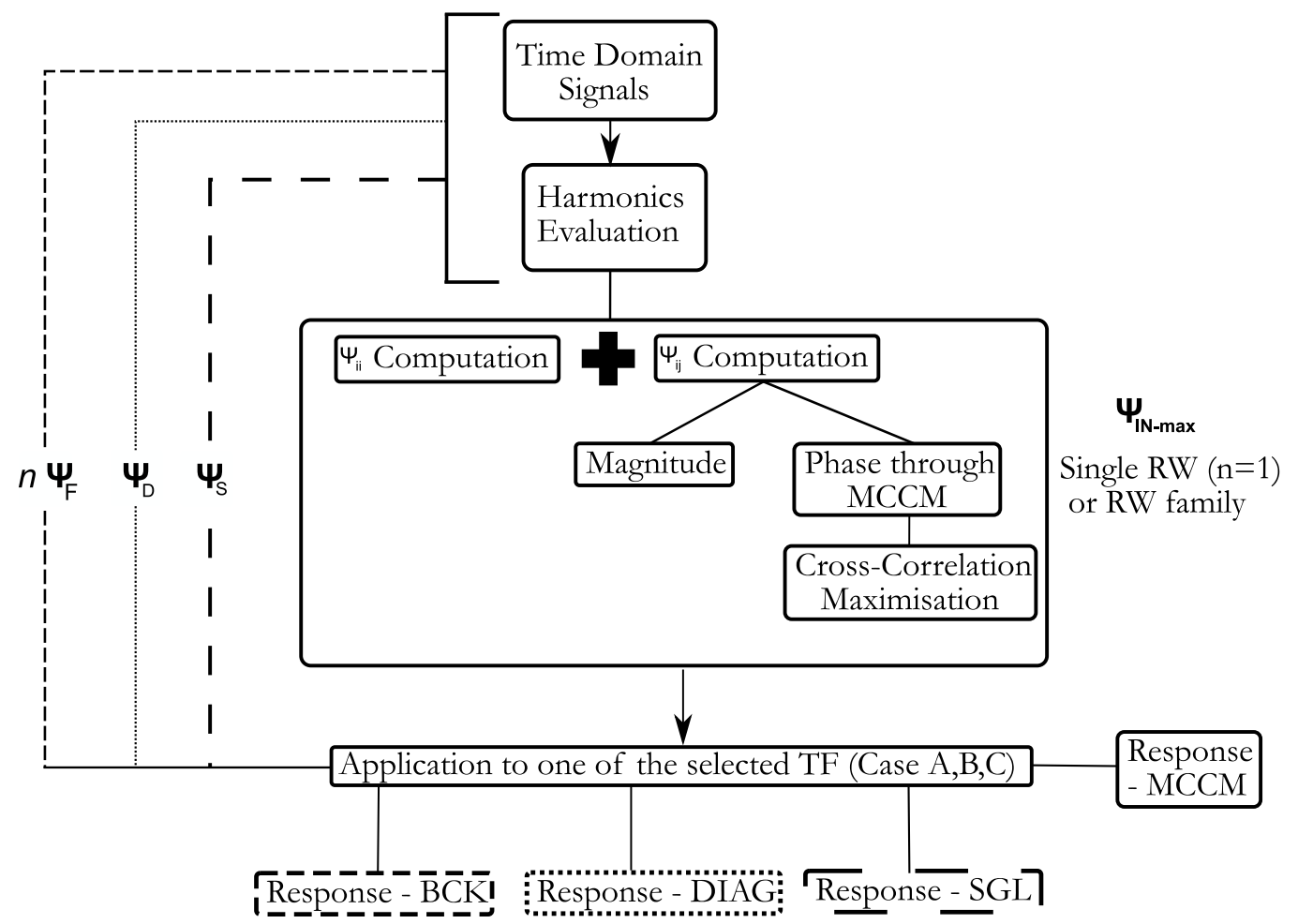

Figure 4: Flow chart of the process for $M C C M$ validation and comparison. From the first two blocks (Time Domain Signals and Harmonics Evaluation) the different disturbance input matrices are evaluated (the big block summarises the $M C C M$ procedure). These are then fed into the transfer function block and the responses are evaluated.

slightly lower than the common value 3, [41]. Once the frequency vector of peaks $f_{P}$ has been identified and then normalised, it is then checked against the $\underline{h_{P}}$ identified at the other wheel speeds $\omega_{2, \ldots, U}$, where $\omega_{U}$ is the highest measured rotational speed to identify consistency all over the velocity range. Finally, a binning procedure is applied: if $h_{P i}$ appears at least in $30 \%$ of the available sets of speed $\underline{\omega}$, it is saved in the harmonics vector $\underline{h}_{P}^{*}$, otherwise discarded.

These steps are followed for both disturbances $F_{X}(t)$ and $F_{Y}(t)$. The two harmonic vectors obtained are then combined to get the radial force harmonics of the RW. The methodology has been further developed by checking the behaviour of the spectral coherence $\xi_{i j}(f)$ at the harmonic locations. This parameter plays a fundamental role in the definition of harmonics linking the 


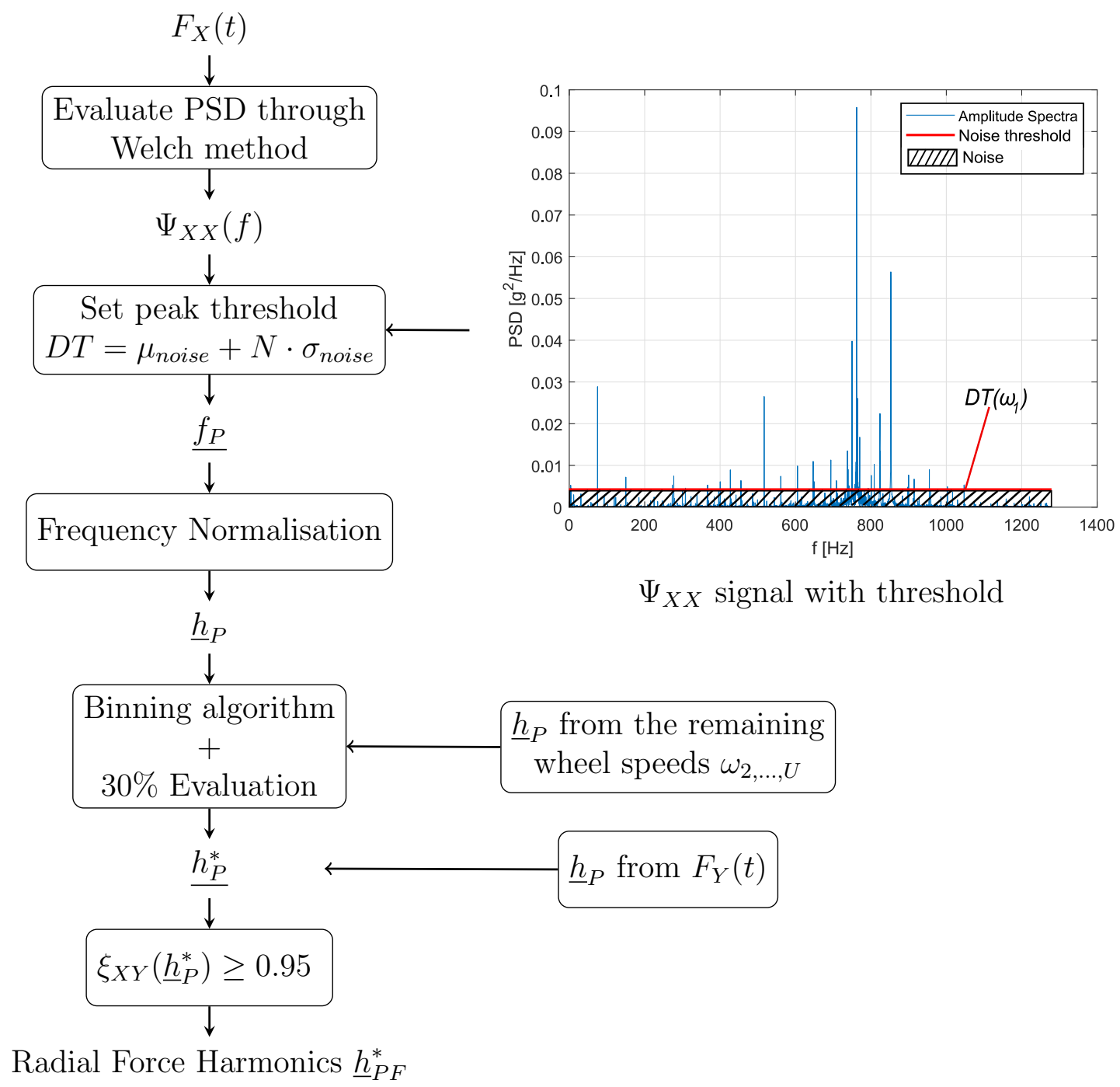

Figure 5: Steps for Harmonics Identification at wheel speed $\omega_{1}$

$\Psi_{i j}$ magnitude with $\Psi_{i i}$ and $\Psi_{j j}$ on the $\underline{f_{P}}$ vector as follows:

$$
\xi_{i j}(f)=\frac{\left|\Psi_{i j}(f)\right|^{2}}{\left|\Psi_{i i}(f)\right| \cdot\left|\Psi_{j j}(f)\right|}
$$

It can range between 0 (uncorrelated signals) and 1 (linearly dependent signals), [43]. In the case of an harmonic, it can be shown that $\xi_{i j} \sim 1$, as a 
physical phenomenon is occuring inside the wheel (imperfection on the ball bearing or manufacturing defect on the cage,...), [44]. By looking at the radial force harmonics, the spectral coherence $\xi_{X Y}\left(\underline{h}_{P}^{*}\right)$ is evaluated at the harmonics identified. As already underlined, RW harmonics represent vibrations inside the wheel, due to imperfections or manufacturing tolerances. This means that the generated disturbances at these specific locations show a spectral coherence function $\xi_{X Y}(f)$ close to 1 (>0.95). This further check is performed and the harmonics whose $\xi$ value is below the threshold $(\xi<0.95)$ are discarded. The process is iterated for axial force using $F_{Z}(t)$, and radial torque harmonics, using $M_{X}(t)$ and $M_{Y}(t)$.

\subsection{Evaluation of $P S D$ and CPSD for a $R W$}

Having explained the two main theoretical pillars of this study, i.e. RW harmonics and PSD/CPSD meaning, these are now combined in the following example for a more realistic case. The goal of this example is to show that the previous findings about directional and rotational disturbances can also be applied in the case of a real RW.

A RW is considered at constant rotational speed $\omega$ and its generated disturbances are measured in the time domain using a dynamometric platform. RW blocked disturbances are then rigidly applied to the structure under analysis, which is the panel described in the previous example. Once all the terms of the input matrix $\boldsymbol{\Psi}_{\mathbf{I N}}$ are evaluated from the time domain through the Welch periodogram, Eq. 3 is used to estimate the reaction force $R_{Z}$ on the panel. In particular, two input matrices $\Psi_{\text {IN }}$ are considered: the fully populated matrix, $\boldsymbol{\Psi}_{\mathbf{I N}-\mathbf{F}}$, and the diagonal $\boldsymbol{\Psi}_{\mathbf{I N}-\mathbf{D}}$, see Appendix A. They are then plugged into Eq. 3 to evaluate $R_{Z}$ (note that the transfer function matrix $\mathbf{H}$ is the same as the one in Section 2.2).

Figure 6 shows the results for the main imbalance and for a generic harmonic. It can be observed that in the first case no variation in the response is recorded between $\boldsymbol{\Psi}_{\mathbf{I N}-\mathbf{F}}$ and $\boldsymbol{\Psi}_{\mathbf{I N}-\mathbf{D}}$ cases. This is due to the nature of this imbalance. Indeed, Figure 7 shows the phase shift between reaction wheel disturbances along directions $\mathrm{X}$ and $\mathrm{Y}$ for the main imbalance $(h=1)$ at different wheel speeds. For each wheel speed, three measurements of phases are available: the phase content oscillates around an average value of $88.2^{\circ}$ that can be approximated to $90^{\circ}$. Hence it can be deduced that this harmonic

exhibits a rotational behaviour. This rotational feature justifies the lack of differences between the responses for the cases $\boldsymbol{\Psi}_{\mathbf{I N}-\mathbf{F}}$ and $\boldsymbol{\Psi}_{\mathbf{I N}-\mathbf{D}}$ shown in Figure 6a, in line with the findings in Section 2.2. Conversely, other har- 


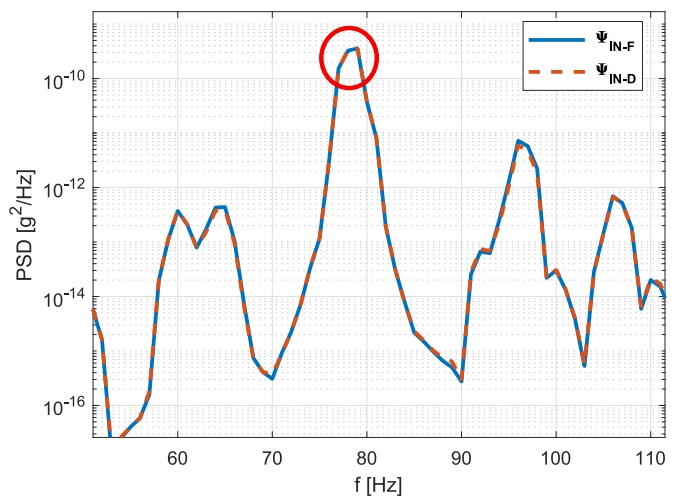

(a) Main imbalance

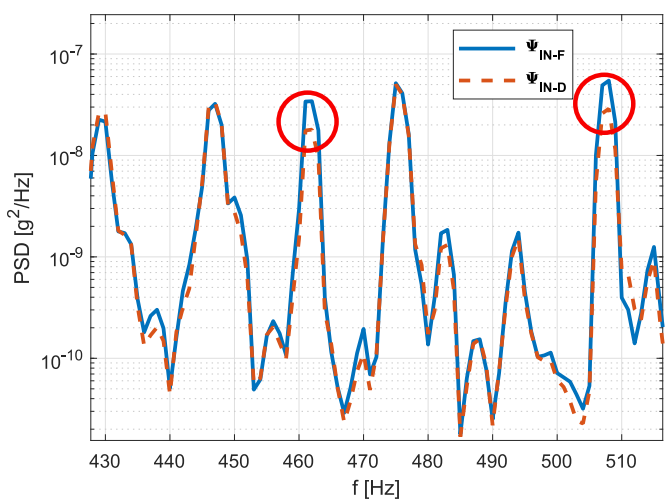

(b) Generic harmonic

Figure 6: Evaluation of the reaction $R_{Z}$ at different harmonic locations in the 2 cases of $\Psi_{\text {IN }}$

monics, such as the ones highlighted in Figure 6b, exhibit a non rotational behaviour, hence significant difference in the two cases, proving the relevance of the $\Psi_{i j}$ terms. Thus, when a RW has to be characterised through spectral

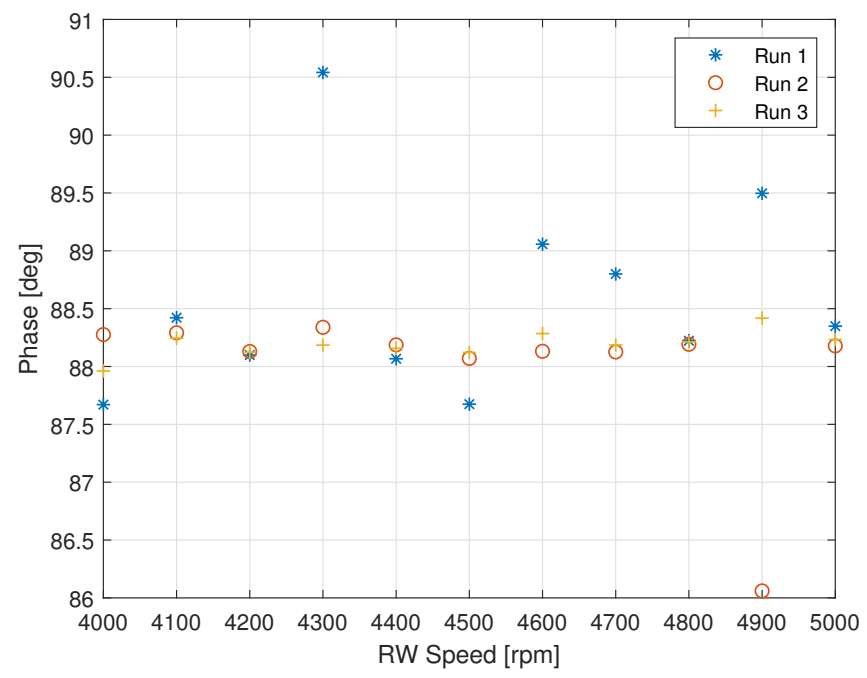

Figure 7: Phase shift between disturbance along $\mathrm{X}$ and $\mathrm{Y}$ directions for 3 different measurements

input matrix $\Psi_{\text {IN }}$ in frequency domain, both diagonal and off diagonal terms 
need to be considered.

\subsection{Single $R W-\Psi_{\mathbf{I N}}(f)$ Identification}

Having shown the relevance of $\Psi_{i j}$ terms in vibration predictions, this achievement is implemented to build $\boldsymbol{\Psi}_{\mathbf{I N}-\mathbf{m a x}}$, referred to as generalised disturbance input matrix corresponding to the largest disturbance produced by a family of $n$ RWs. The task is complex mainly for two reasons: first, the effects produced by $\boldsymbol{\Psi}_{\mathbf{I N}-\text { max }}$ are generally dependent on the transfer function considered, via Eq. 3. Secondly, this matrix contains complex terms. To overcome these limitations and build $\boldsymbol{\Psi}_{\mathbf{I N}-\mathbf{m a x}}$, the case of a single RW is considered as first step (which represents the limit case of a family with $n=1$ ). Here, the diagonal terms of the matrix are obtained using the exact procedure, i.e. starting from the time domain signals, the modified Welch periodogram is applied and $\Psi_{i i}(f)$ are computed. Then, in order to characterise $\Psi_{i j}(f)$ terms and populate the off-diagonal elements, a more elaborated procedure is followed. Indeed, as already explained, the offdiagonal components are influential at the harmonics, see definition of $\xi_{i j}(f)$ in Eq. 7. Since RW harmonics represent a physical phenomenon occurring inside the RW, their frequency contents meet the following condition:

$$
f_{m} \mid \xi_{i j}\left(f_{m}\right) \geq 0.95
$$

Once the $m$ values meeting Condition 8 are identified, Eq. 7 is rearranged to obtain $\left|\Psi_{i j}\left(f_{m}\right)\right|$. Then, the phase $\phi_{i j}\left(f_{m}\right)$ associated to $\Psi_{i j}\left(f_{m}\right)$ has to be estimated. The evaluation of this parameter is usually driven by the interaction with the structure the input is applied to. Indeed, the phase dictates how $\Psi_{i j}\left(f_{m}\right)$ magnitude is split between real and imaginary part and how both components interact with the transfer function of the structure. The aim of the proposed methodology involves the evaluation of the input source by simply working on the source itself, regardless of the considered structure. As such, the phase is evaluated through the Cross Correlation $C_{i j}(t)$ in time domain. In fact, the Fourier transform of this quantity is, by definition, equal to the Cross Power Spectral Density, [36]. The main innovation of the proposed method relies on the phase evaluation $\phi_{i j}\left(f_{m}\right)$, as it is directly driven by the Cross Correlation. In particular, this methodology aims at finding the physical scenario where this quantity is maximised in time.

Searching the maximum of $C_{i j}(t)$ corresponds to the physical condition where the combination of the considered signals $i$ and $j$ is maximised. In 


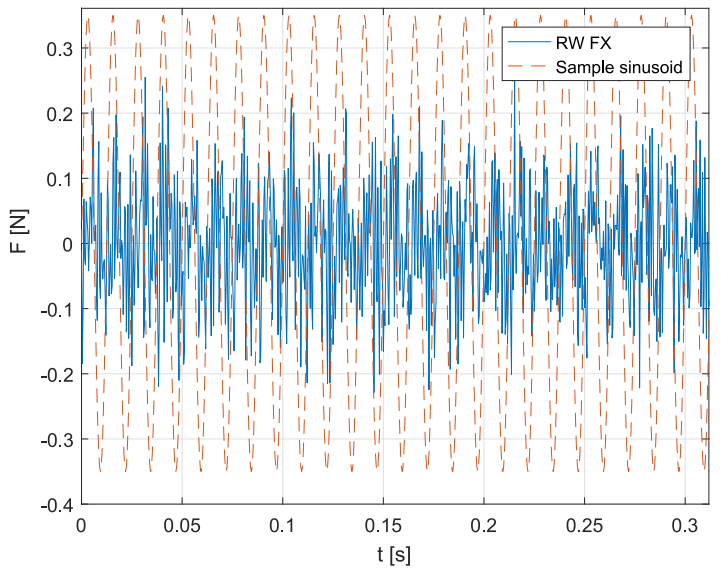

$F_{X}(t)$ - Example of sinusoid filter at $f_{m}=80 \mathrm{~Hz}$

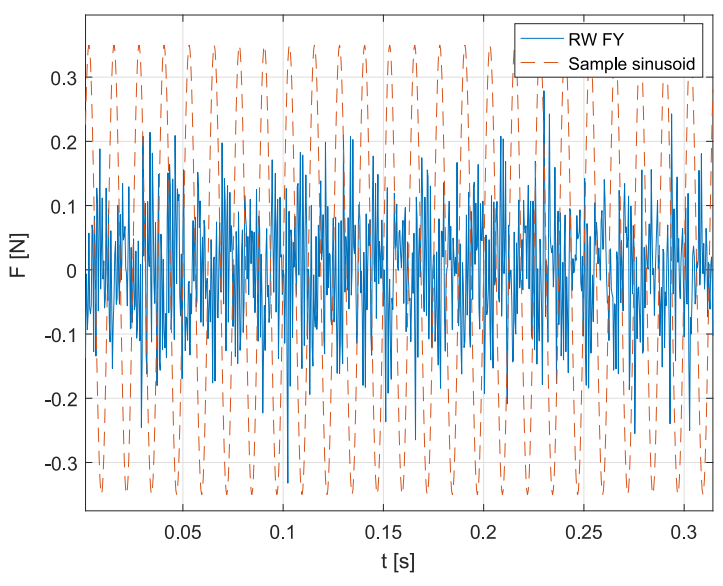

$F_{Y}(t)$ - Example of sinusoid

filter at $f_{m}=80 \mathrm{~Hz}$

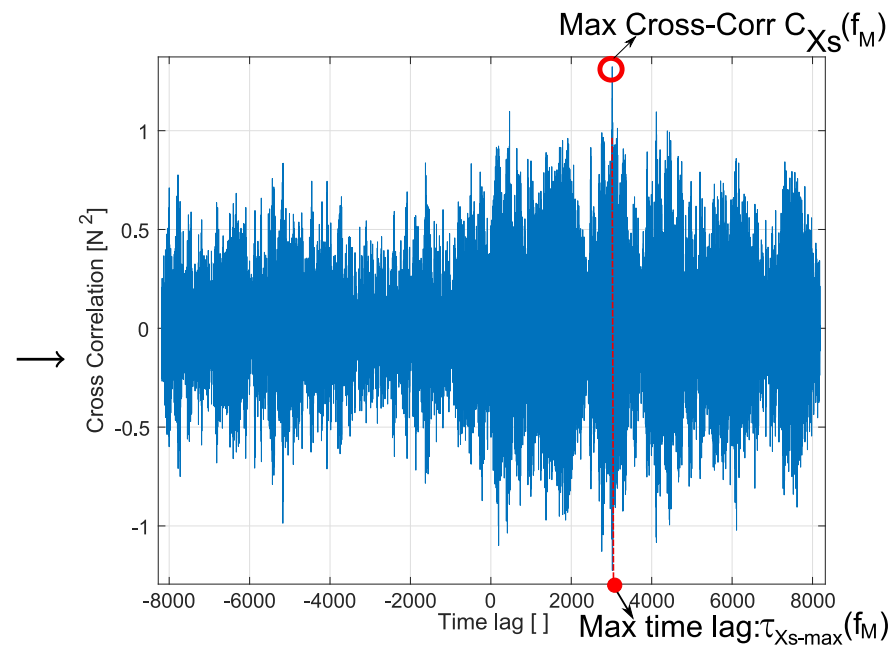

Cross-Correlation $C_{X s}\left(t, f_{m}\right)$

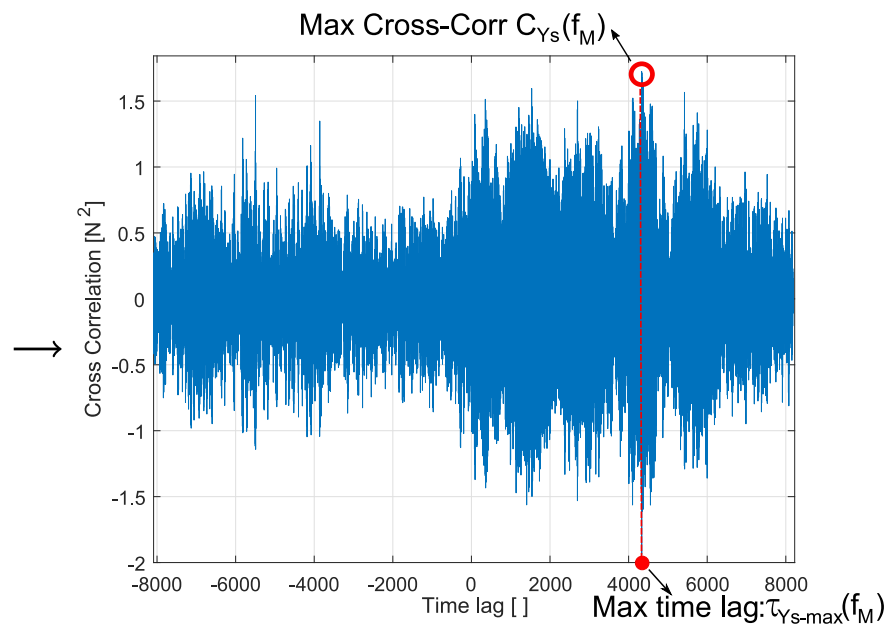

Cross-Correlation $C_{Y s}\left(t, f_{m}\right)$

Figure 8: $\phi_{X Y-\max }(f)$ estimation: evaluation of the maximum cross-correlation between signal $F_{X}(t)$ (and $\left.F_{Y}(t)\right)$ and $s\left(t, f_{m}\right)$ with $f_{m}=80 \mathrm{~Hz}$. The procedure is iterated $K$ times

particular, this maximisation occurs at $m$ frequency steps satisfying Condition 8. The method will be referred to as Maximum Cross Correlation 
Method (MCCM). The main advantage of MCCM stays in the independence on the structure it is applied to, as it purely works on the source. As already mentioned, one of the main issues of the available methodologies is the variability of their performances depending on the structure it is applied to. As it will be shown in this paper, MCCM proves to be robust for applications to structures with different properties, geometries and complexities. In this way, it is possible to determine such disturbance input matrix in a generic case.

In the following, the methodology will be explained in details for the generation of the term $\Psi_{X Y}\left(f_{m}\right)$. Consider a RW and its two associated disturbances $F_{X}(t)$ and $F_{Y}(t)$ obtained through dynamometric table measurements. They generate the term $\Psi_{X Y}\left(f_{m}\right)$ of the generic disturbance input matrix. This term is defined through its magnitude $\left|\Psi_{X Y}\left(f_{m}\right)\right|$ and phase $\phi_{X Y-\max }\left(f_{m}\right)$. The phase contains the subscript $-\max$ as it is obtained from the maximisation of the Cross Correlation. In order to obtain the phase information at each frequency considered, the disturbance signal content at each frequency step is needed. This is done introducing a series of sample sinusoids $s\left(t, f_{m}\right)$ as shown in Figure 8 . These work as a filter for the signal $F_{X}(t)$ and $F_{Y}(t)$ and are represented by sine waves resonating at each of the $m$ frequency steps considered in the analysis on a time vector $t$ which comes from the measurements. For each sinusoid $s\left(t, f_{m}\right)$, the Cross Correlation between signal $s\left(t, f_{m}\right)$ and $F_{X}(t)$ is evaluated. $C_{X s}(\tau)$ is then maximised to get $C_{X s}\left(\tau=\tau_{\max -X s}\right)$ where $\tau_{\max -X s}$ is the time lag at which the $C_{X s}$ is maximised, see Figure 8. The same procedure is followed for the signal $F_{Y}(t)$ and $\tau_{\max -Y s}\left(f_{m}\right)$ is calculated. Eventually the difference between the two time lags, $\tau_{\max -X s}\left(f_{m}\right)$ and $\tau_{\max -Y s}\left(f_{m}\right)$ is computed to obtain the time lag $\tau_{X Y}\left(f_{m}\right)$ where the cross-correlation between $F_{X}(t)$ and $F_{Y}(t)$ is maximised for that specific frequency content at $f_{m}$. Finally, the phase $\phi_{X Y-\max }\left(f_{m}\right)$ is obtained as:

$$
\phi_{X Y-\max }\left(f_{m}\right)=2 \pi \cdot \Delta t \cdot\left(\tau_{\max -X s}\left(f_{m}\right)-\tau_{\max -Y s}\left(f_{m}\right)\right) \cdot f_{m}
$$

where $\Delta t$ is the time interval between two subsequent measurements from the dynamometric table. Having computed the magnitude $\Psi_{X Y}\left(f_{m}\right)$ and the phase $\phi_{X Y-\max }\left(f_{m}\right)$, the term $\Psi_{I N-\max }\left(1,2, f_{m}\right)$ has been fully defined. Once $\phi_{X Y-\max }\left(f_{m}\right)$ has been determined, the same approach is applied to obtain $\phi_{X Z-\max }\left(f_{m}\right)$ and for the torques $\phi_{X M X-\max }\left(f_{m}\right)$ and $\phi_{X M Z-\max }\left(f_{m}\right)$. The remaining phases are calculated through time constraints, i.e. $\tau_{\max , M Z Z}\left(f_{m}\right)=$ 
$\tau_{\max , M Z X}\left(f_{m}\right)-\tau_{\max , X Z}\left(f_{m}\right)$. With $M C C M$ it is then possible to fully populate the disturbance matrix $\boldsymbol{\Psi}_{\mathbf{I N}-\max }(f)$. The methodology, however, finds its best application in the case of multiple RWs, as shown in the following paragraph.

\subsection{Multiple RWs Case}

If $n$ nominally identical RWs are available, these identify a family of sources, i.e. devices with the nominally identical components (such as bearings). Because of manufacturing defects and geometric tolerances these wheels can show differences in the amplitude of the generated disturbances. This is seen in Figure 9 where the blocked disturbances $F_{X}(t)$ and $F_{Y}(t)$ are

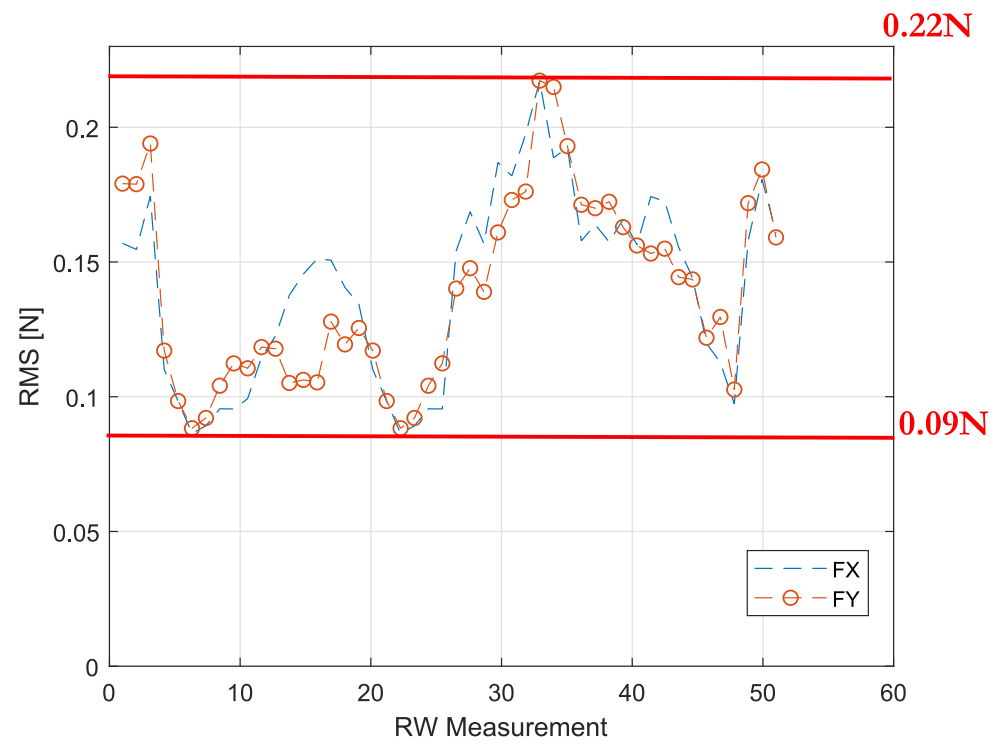

Figure 9: RMS of the measured disturbances $F_{X}(t)$ and $F_{Y}(t)$ in blocked configuration for 51 nominally identically RWs at fixed speed $\omega$

considered for 51 nominally identical RWs. RMS value is shown to oscillate between $0.09 \mathrm{~N}$ and $0.22 \mathrm{~N}$. Similarly, significant variations in terms of amplitudes are observed in the frequency domain, Figure 10.

Hence, minimal defects inside the wheel can alter the signal, generating a broad band of amplitudes. In the case of a family of RWs, $M C C M$ finds its best application as it enables the calculation of a single disturbance input matrix $\Psi_{\mathbf{I N}-\max }$ which will be referred to as generalised disturbance input 


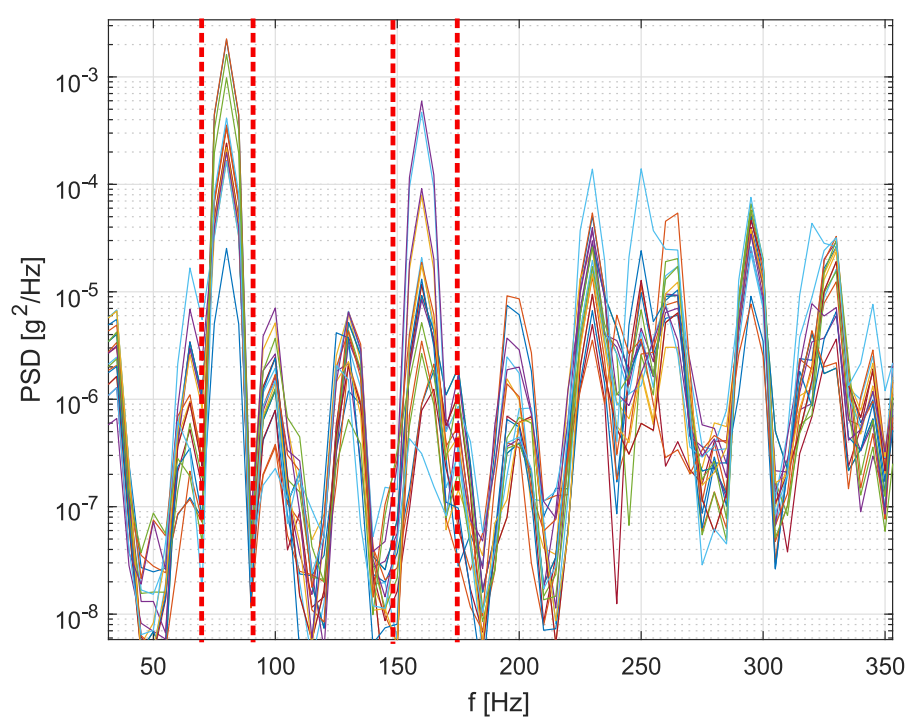

Figure 10: Frequency domain representation of the disturbance $F_{X}$ for nominally identical RWs - Low frequency

matrix, able to envelope the effects produced by the whole family of sources. In this case, the diagonal elements of the matrix $\Psi_{\mathbf{I N}-\max }$ are expressed as follows:

$$
\Psi_{I N-i i-\max }\left(f_{m}\right)=\max _{n}\left[\Psi_{I N-i i}\left(n, f_{m}\right)\right]
$$

As far as $\Psi_{i j}$ terms are concerned, it can happen that, because of inner defects inside the RW, a specific frequency value $f_{m}$, representing an harmonic, shows that condition 8 is satisfied for a number of wheel $K$, with $K<n$. In other words, not all the source devices of the same family show exactly the same harmonics. In particular $K$ varies depending on the harmonic considered and it is automatically calculated through $\xi_{i j}\left(f_{m}\right)$. In this case, the off-diagonal term $\Psi_{I N-i j-\max }$ is evaluated as:

$$
\left|\Psi_{I N-i j-\max }\left(f_{m}\right)\right|=\sqrt{\overline{\xi_{i j}}\left(f_{m}\right) \cdot\left|\max _{K}\left[\Psi_{I N-i i}\left(K, f_{m}\right)\right]\right| \cdot\left|\max _{K}\left[\Psi_{I N-j j}\left(K, f_{m}\right)\right]\right|}
$$

where the average $\overline{\xi_{i j}}\left(f_{m}\right)$ is computed over $K$ RWs. If condition 8 is not met, then $\xi_{i j}\left(f_{m}\right)=0 \rightarrow \Psi_{I N-i j-\max }\left(f_{m}\right)=0$.

For the phase estimation, the procedure described in Section 2.6 is iterated 
$K$ times to compute the phase $\phi_{i j}\left(f_{m}\right)$. Considering the signals $F_{X}(K, t)$ and $F_{Y}(K, t)$ as an example, $K$ values of $\phi_{X Y}\left(f_{m}\right)$ are obtained and are then averaged to get $\phi_{X Y-\max }\left(f_{m}\right)$. The same procedure is followed for the remaining phases. The methodology is iterated at all frequency values meeting condition 8.

\section{Application}

\subsection{RW Data Set and Benchmark Method}

Before focusing on the application, the used data set is defined. It consists of 30 RWs from the same family/batch. The wheels are 100 SP-O produced by Surrey Satellite Technology Limited (SSTL) and they are used to provide highly agile attitude control for Earth Observation purposes. The measurements have been obtained in a blocked configuration, using a dynamometric table, with the wheel rigidly connected to it. For each RW, a ramp-up test was run from 100 to $5000 \mathrm{rpm}$, taking measurements every $100 \mathrm{rpm}$ (50 measurements in total) and sampling rate of $2560 \mathrm{~Hz}$. A benchmark method $(B C K)$ is generated, which represents the real scenario of the RW family. This is created by characterising the 30 RWs separately, hence the full disturbance input matrix $\mathbf{\Psi}_{\mathbf{F}}$ for each wheel is fully populated from the time domain signals using the Welch method. Each of the $30 \mathbf{\Psi}_{\mathbf{F}}$ matrices is assessed separately on the structure under test using Eq. 3 and, once the transfer function matrix $\mathbf{H}$ is evaluated, six responses are obtained. These are then maximised at each frequency step in order to obtain the maximal responses $\underline{\Psi}_{O U T-\max }$ representing the benchmark method. Since the proposed methodology aims at describing the family of RW on a generic structure, the goal is to show that $\boldsymbol{\Psi}_{\mathbf{I N}-\max }$ generates a response as close as possible to $\underline{\Psi}_{O U T-\max }$.

Before the evaluation of the responses, the radial harmonics lines are identified from the Campbell plot in Figure 11 and compared with the computed ones using the methodology explained in Section 2.4. Results show an average difference of the harmonic coefficients of less than $1 \%$. The procedure has been reiterated for the set of $30 \mathrm{RW}$ measurements. The following pages of this article compare $M C C M$ with other two methods. A constant wheel speed $\omega$ is assumed. These methods are defined as follows:

- Diagonal input $\left(D I A G, \mathbf{\Psi}_{\mathbf{D}}\right)$ : this is the procedure currently adopted in literature where $n$ RWs are used to obtain the generalised input 


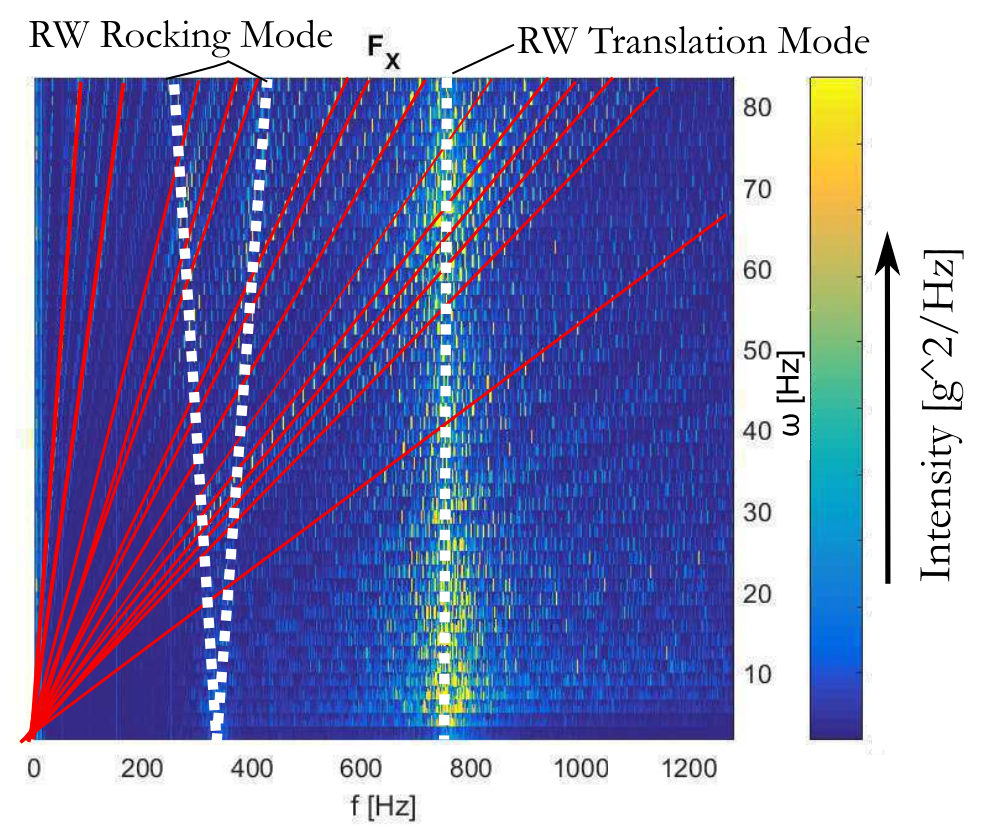

Figure 11: Campbell plot for one of the 30 RWs considered: identification of the radial harmonics (red lines). The rocking mode $(330 \mathrm{~Hz})$ and the translational mode $(760 \mathrm{~Hz})$ are also visible

matrix $\Psi_{\mathbf{I N}-\mathbf{D}}$ containing the diagonal terms only. These are obtained as maximum of the single $n$ diagonal terms, as described in the first part of Section 2.6.

- Single term $\left(S G L, \mathbf{\Psi}_{\mathbf{S}}\right)$ : in this case the input is provided as a single term from the diagonal of the matrix in DIAG. Each of them generates a response vector along six directions, i.e.:

$$
\begin{gathered}
\boldsymbol{\Psi}_{\text {OUT-X}}(f)=\mathbf{H}(\mathbf{f}) \cdot \boldsymbol{\Psi}_{S-X}(f) \cdot \mathbf{H}^{H}(f) \\
\boldsymbol{\Psi}_{S-X}(f)=\left[\begin{array}{cccc}
P S D_{x x} & 0 & \ldots & \ldots \\
0 & 0 & \ldots & \ldots \\
\ldots & 0 & \ldots & \ldots
\end{array}\right]
\end{gathered}
$$




$$
\vec{\Psi}_{\text {OUT-X }}(f)=\operatorname{diag}\left(\Psi_{\text {OUT-X}}(f)\right)=\left[\begin{array}{c}
\Psi_{O U T-X X}(f) \\
\Psi_{O U T-Y X}(f) \\
\Psi_{O U T-Z X}(f) \\
\Psi_{O U T-M X X}(f) \\
\Psi_{O U T-M Y X}(f) \\
\Psi_{O U T-M Z X}(f)
\end{array}\right]
$$

where the response $\Psi_{O U T-i X}(f)$ is computed along direction $i$ due to $\Psi_{S-X}(f)$. Once all the contributions have been evaluated, the final responses are estimated as follows:

$$
\Psi_{\text {OUT-J }}(f)=\sqrt{\sum_{i=1}^{6} \Psi_{\text {OUT-Ji }}^{2}(f)}
$$

where $J$ identifies the direction of the response under evaluation and $i$ the direction considered. This procedure is typically applied for evaluation of microvibration sources on structures as it is highly conservative and ensures high margin of safety during the design stage.

In the next paragraph the results are shown for the case $n=30$, i.e. family of RWs. The full methodology including harmonics identification and $M C C M$ is applied to different structural configurations to assess its validity. In particular the RW is coupled to 3 different supporting structures, each of them with a different level of complexity. The different structures are referred to as:

- Case A: Structural panel

- Case B: Box structure

- Case C: Real spacecraft structure (platform SSTL - 300)

- Locations $I_{1}-O_{1}$

- Locations $\mathrm{I}_{2}-\mathrm{O}_{2}$

In Case $\mathrm{C}$ two different input $\left(I_{1}, I_{2}\right)$ and output locations $\left(O_{1}, O_{2}\right)$ are considered. 


\begin{tabular}{cc}
\hline Property & Value \\
\hline Dimensions $(\mathrm{X} \times \mathrm{Y} \times \mathrm{Z})[\mathrm{m}]$ & $5 \times 2 \times 0.08$ \\
Young modulus $[\mathrm{GPa}]$ & 69.9 \\
Density $\left[\mathrm{kg} / \mathrm{m}^{3}\right]$ & 2810 \\
Poisson ratio [ ] & 0.33 \\
Boundary conditions & Blocked at the 4 nodes \\
\hline
\end{tabular}

Table 3: Case A - Structural panel properties

\subsection{Case A: Structural Panel $(n=30)$}

As first case study, a 2D panel is considered, whose physical properties are reported in Table 3. The reason for choosing this specific structure is that its structural modes do not interact with the harmonics of the RW at the roational speed $\omega$ under analysis, as shown in Figure 12. This example is useful to show purely the effects of $M C C M$ in the prediction of microvibrations compared to $D I A G$ and $S G L$. In addition, various mounting configurations

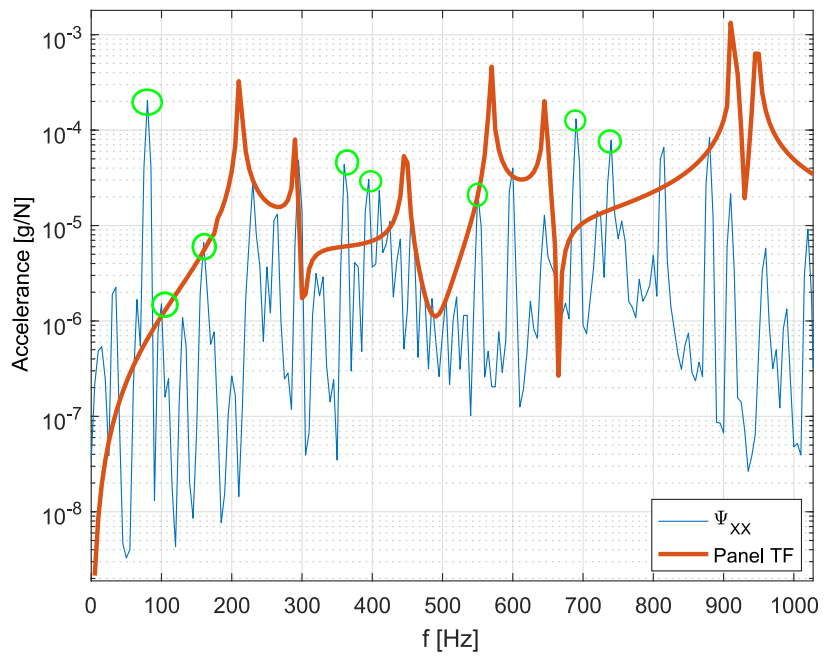

Figure 12: RW harmonics (green circles) do not interact with the modes of the structural panel. Note that there are some $\Psi_{X X}$ peaks that overlap with the structural modes of the panel but they do not represent harmonics as they are not consistent all over the range of RW rotational speed $\omega_{1}, \ldots, \omega_{U}$ considered

of the RW are taken into account: the RW input forces are assigned to the 
structure using a slightly different expression of Eq. 3, which is:

$$
\boldsymbol{\Psi}_{\text {out }}=\mathbf{H} \cdot \mathbf{R} \cdot \Psi_{\mathbf{I N}-\max } \cdot \mathbf{R}^{T} \cdot \mathbf{H}^{H}
$$

where $\mathbf{R}$ is a rotational matrix representing a series of rotations of the RW axes with respect to the reference axes of the structure. In fact, RWs are generally mounted on the $\mathrm{S} / \mathrm{C}$ in different configurations in terms of relative angle with respect to the satellite, [45], for a matter of power consumption and torque requirements. Figure 13 shows the RW frame $\left(X_{R W}, Y_{R W}, Z_{R W}\right)$ and the structure one $\left(X_{S}, Y_{S}, Z_{S}\right)$. Matrix $\mathbf{R}$ describes the rotation between

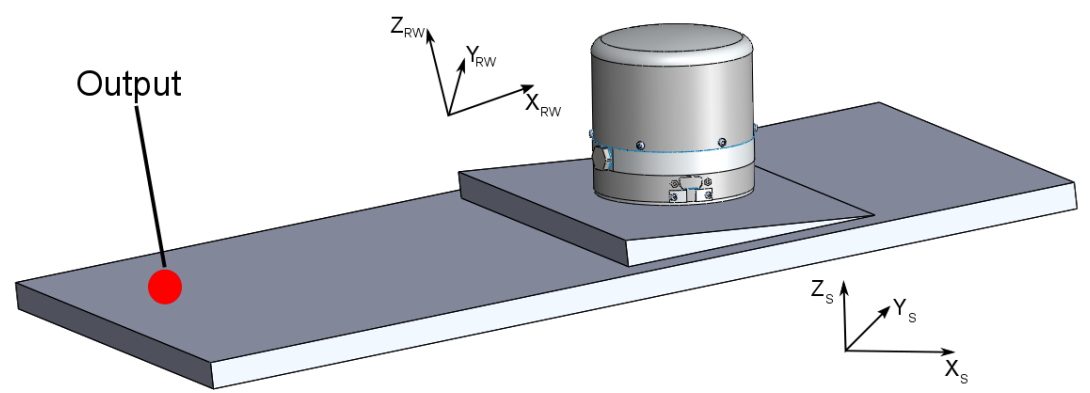

Figure 13: Mounting configuration of the RW on the structure

the two reference frames. They can also be combined as a series of consequent rotations. In the verification of $M C C M$ a random selection of rotation angles $\left[-180^{\circ} 180^{\circ}\right]$ and rotation axes has been used, in order to generate random and uncorrelated simulations. The disturbance input matrix $\boldsymbol{\Psi}_{\mathbf{I N}-\max }$ has been applied to the panel in different configurations in terms of rotation. The results are reported in Table 4 . Here the ratio between the two considered cases is shown at different harmonics. These values are the average results computed on 15 different simulations, i.e. different rotation configurations. Looking at the results along $X$ direction, Table 4 , it can be seen that the prediction factor is closer to 1 for the $M C C M-B C K$ than the other two (the aim would be to obtain coefficient 1 at all the considered harmonics). In particular, the $S G L$ method shows the largest variations compared to $B C K$. These results are due to the assumption that the input is applied separately for each direction, refer to Eq. 13 for its definition, whereas in the reality all the directions contribute together. Looking at DIAG, the fundamental harmonic, which has been shown as rotational, exhibits a very high factor with respect to $B C K$, i.e. 2.16. This result could appear in 


\begin{tabular}{cccc}
\hline Harmonic number & Ratio btw $M C C M-B C K$ & Ratio btw $D I A G-B C K$ & Ratio btw $S G L-B C K$ \\
\hline 1.02 & 1.00 & 2.16 & 4.6 \\
1.23 & 1.29 & 1.37 & 2.7 \\
2 & 1.06 & 1.52 & 6.6 \\
3.91 & 1.3 & 1.7 & 1.2 \\
4.51 & 1.16 & 1.58 & 11 \\
4.87 & 1.05 & 1.29 & 3.2 \\
6.89 & 1.57 & 1.63 & 1.8 \\
8.63 & 1.7 & 3.1 & 4.5 \\
9.25 & 1.3 & 1.9 & 4.31 \\
Average & 1.27 & 1.81 & 4.4 \\
\hline
\end{tabular}

Table 4: Case $A$ - Comparison of responses along $X$ : each value is the average of 15 different configurations. Values indicate the ratio between the two considered responses at $\omega_{R W}=4800 \mathrm{rpm}$

contrast with the statement about the main imbalance in Section 2.5, i.e. $\Psi_{i j}$ are irrelevant for rotational harmonics. In reality, Section 2.5 was assuming an in plane problem with $\Psi_{\mathbf{I N}-\max }(f) \in \mathbb{C}^{2 x 2}$ where $\Psi_{i j}$ have shown to be irrelevant. Conversely, in the current example, the full matrix is generated, hence $\boldsymbol{\Psi}_{\mathbf{I N}-\max }(f) \in \mathbb{C}^{6 x 6}$. This implies that all the $\Psi_{i j-\max }(f)$ terms can affect the response, not only the rotational $\Psi_{X Y}(f)$ as for the example in Section 2.5.

\subsection{Case B: Box Structure $(n=30)$}

In this second example a more complex structure, which is a box structure, is examined. A rigid connection is generated at the top surface of the structure where the unitary excitation force is applied to evaluate the transfer function matrix $\mathbf{H}$. The structure is fixed to the ground at the 4 vertices, as shown in Figure 14. The modes until $800 \mathrm{~Hz}$ are reported in Table 5. Results for this case are shown in Table 6 in which acceleration responses

\begin{tabular}{lccccc}
\hline Mode number & 1 & 2 & 3 & 4 & 5 \\
Frequency $[\mathrm{Hz}]$ & 250 & 355 & 465 & 505 & 640 \\
\hline
\end{tabular}

Table 5: Box structure modes (up to $800 \mathrm{~Hz}$ )

along $Y$ direction are considered. These findings are in line with the results from the previous case. $M C C M$ is able to provide more accurate estimations compared to $D I A G$ and $S G L$. An uncertainty factor save of 0.5 is obtained with respect to $D I A G$. In addition, one of the vibration modes of 
the structure interacts with one of the considered harmonics, i.e. 4.51. This is known to be a critical load case in RW characterisation because of the RW signature amplification. Indeed, currently adopted models tend to neglect the experimental data coupled with RW modes. Conversely, $M C C M$ shows good agreement also in this situation compared to DIAG and SGL methods.

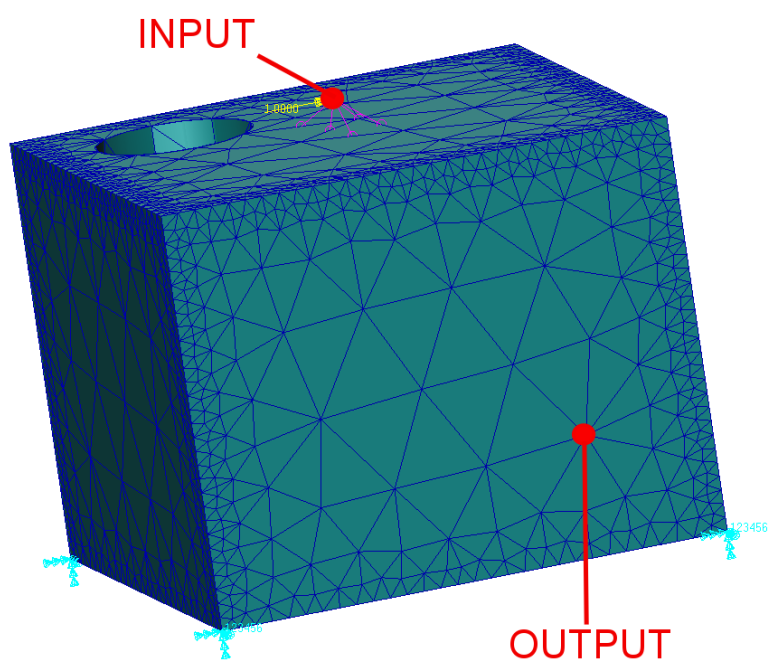

Figure 14: FEM of the box structure

\begin{tabular}{cccc}
\hline Harmonic number & Ratio btw $M C C M-B C K$ & Ratio btw $D I A G-B C K$ & Ratio btw $S G L-B C K$ \\
\hline 1.02 & 1 & 1.86 & 13 \\
1.23 & 1.33 & 1.58 & 2.48 \\
2 & 1.2 & 1.4 & 1.6 \\
3.91 & 1.4 & 1.5 & 1.21 \\
$\mathbf{4 . 5 1}$ & 1.01 & 1.45 & 7.43 \\
4.87 & 1.2 & 1.51 & 4.2 \\
6.89 & 1.09 & 1.34 & 1.86 \\
8.63 & 1.23 & 1.6 & $\sim 40$ \\
9.25 & 1.3 & 1.8 & 5.2 \\
Average & 1.2 & 1.55 & 8.55 \\
\hline
\end{tabular}

Table 6: Case $B$ - Comparison of the $Y$ responses: each value is the average of 15 different configurations. Values indicate the ratio between the two considered responses at $\omega_{R W}=$ $4800 \mathrm{rpm}$ 


\subsection{Case C:SSTL-300 ( $n=30)$}

In this last case a real space structure is taken into account. SSTL300 is a platform that can be adopted for a wide range of applications and accommodate a large number of payload and structural configurations. It has already been employed for $D M C-3$ constellation and NigeriaSat -2 . Two

\begin{tabular}{cc}
\hline $\begin{array}{c}\text { Harmonic number } \\
\text { (corresponding frequency }[\mathrm{Hz}])\end{array}$ & $S S T L-300$ main Eigenmodes \\
{$[\mathrm{Hz}]$}
\end{tabular}

Table 7: RW harmonics (at $\omega_{R W}=4800 \mathrm{rpm}$ ) and $S S T L-300$ eigenmodes. Bold values represent the crossing between harmonics and structural vibration modes

transfer functions matrices $\mathbf{H}$ are considered, namely $\mathbf{H}_{\mathbf{1}}$ and $\mathbf{H}_{\mathbf{2}}$. The former assumes as input and output nodes $I_{1}$ and $O_{1}$, representing the location of the RW for the input and imager location for the output. Analogously, $\mathbf{H}_{\mathbf{2}}$ uses input $\mathrm{I}_{2}$ corresponding to a different RW and output $\mathrm{O}_{2}$ for the camera, as shown in Figure 15. Table 8 shows the results of the two sub-cases considered. Similar results are also obtained along direction $Y$ but these have not been reported for the sake of parsimony.

It is worth noticing in this case that most of the harmonics interact with spacecraft modes (bold values), as shown in Table 7 where a limited list of the spacecraft modes is reported. In addition, the predictions obtained through the $S G L$ method provide very high overestimations. Nevertheless, there are some cases, such as the response at the main imbalance along $Y$ direction, where the $S G L$ shows very good agreement with the benchmark case. Conversely, other harmonics, such as 9.25, exhibit a huge discrepancy. In fact, having obtained $S G L$ response from the square root summation of the single responses, high overestimations and low underestimations can be obtained depending on the sign of the single responses. In $S G L$, the responses are 


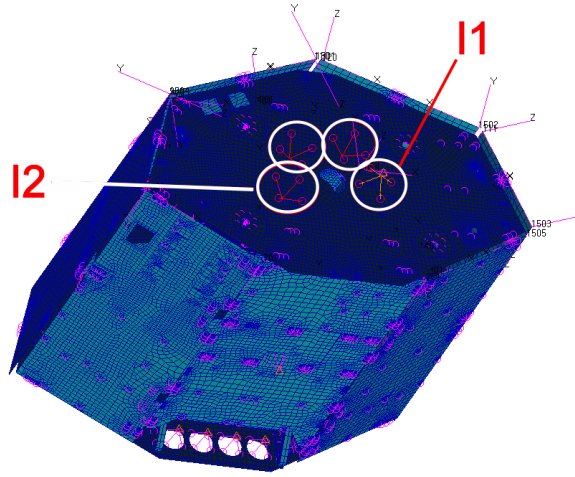

(a) Input locations

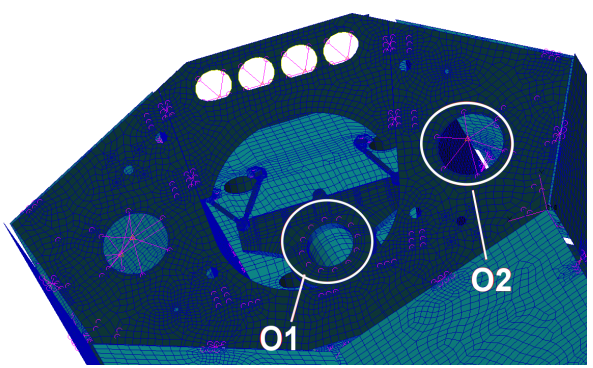

(b) Output locations

Figure 15: SSTL-300 FEM

\begin{tabular}{cccc}
\hline Harmonic number & Ratio btw $M C C M-B C K$ & Ratio btw $D I A G-B C K$ & Ratio btw $S G L-B C K$ \\
\hline 1.02 & 1.26 & 1.92 & 15.83 \\
$\mathbf{1 . 2 3}$ & 1.1 & 1.31 & 5.6 \\
$\mathbf{2}$ & 1.13 & 1.45 & 6.2 \\
$\mathbf{3 . 9 1}$ & 1.61 & 1.58 & 17 \\
$\mathbf{4 . 5 1}$ & 1.04 & 1.11 & 7.43 \\
4.87 & 1.11 & 1.21 & 4.2 \\
$\mathbf{6 . 8 9}$ & 1.2 & 2.57 & 185 \\
8.63 & 1.28 & 1.69 & 5.67 \\
$\mathbf{9 . 2 5}$ & 1.46 & 1.95 & 7.6 \\
Average & 1.24 & 1.64 & $>20$ \\
\hline
\end{tabular}

Table 8: Case $C$ - Comparison of the $X$ responses: each value is the average of 15 different configurations. Average between the results obtained for $\mathbf{H}_{\mathbf{1}}$ and $\mathbf{H}_{\mathbf{2}}$. Values indicate the ratio between the two considered responses at $\omega_{R W}=4800 \mathrm{rpm}$

always considered to be positive, as the square root summation is involved, hence in most of the cases large overshoots are predicted. Nevertheless, there are some situations where the sign of the transfer function matrix $\mathbf{H}$, can lead to some underestimations. Looking at the performance of $M C C M$ in this last case, a safety margin reduction of approximately 0.4 for the $X$ direction response is recorded. Similar results are obtained along direction $Y$.

\section{Identification of the minimum number of RWs: Convergence Analysis}

$M C C M$ has shown to provide very good predictions compared to $D I A G$ and $S G L$. In addition, the methodology reveals a very important property 
that can be used in practical applications, as explained hereafter.

A family of $n=30$ RWs is considered. $M C C M$ has been run assuming a different number of RWs (from $p=1$ to $p=n=30$ ) for each simulation. From Figures 16 it can be concluded that the response converges as $p$ increases. In particular it occurs for all the harmonics at around 20 RWs (i.e. stopping the simulations at $p \geq 20$ RWs gives the same result as $n=30 \mathrm{RWs}$ ). Some harmonics converge even before this value. More convergence plots are reported

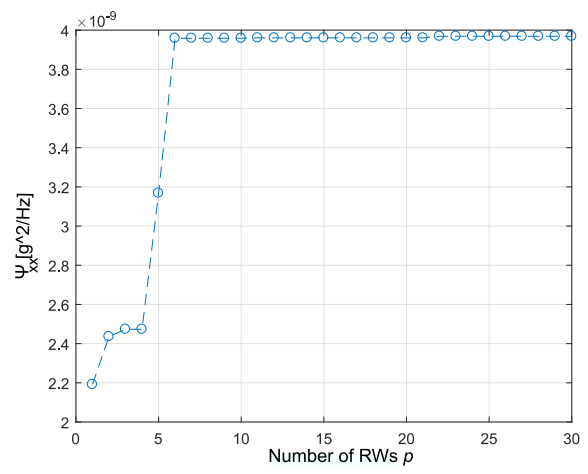

(a) $\boldsymbol{\Psi}_{\mathbf{X X}}$ at $h=1$

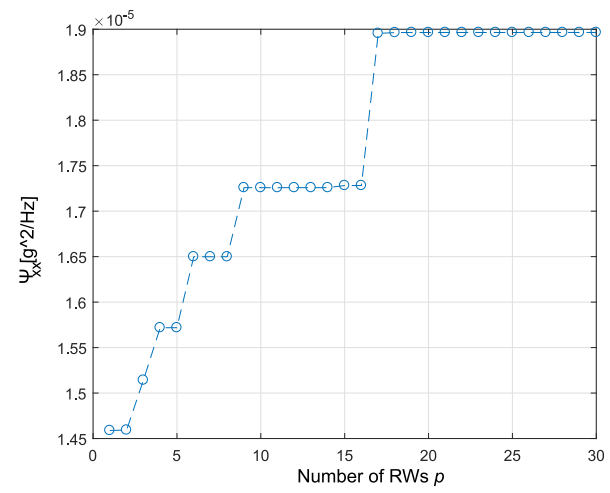

(b) $\boldsymbol{\Psi}_{\mathbf{X X}}$ at $h=3.69$

Figure 16: Convergence of the harmonics $h_{i}$ for response $\boldsymbol{\Psi}_{\mathbf{X X} \text {-OUT }}$ computed on $S S T L-$ 300 using $M C C M$ with $\mathbf{R}=\mathbf{R}_{\mathbf{1}} * \mathbf{R}_{\mathbf{3}}, \theta_{1}=150^{\circ}$ and $\theta_{3}=-104^{\circ}, \mathbf{H}=\mathbf{H}_{\mathbf{1}}$

in Appendix A where other cases are shown as well as the responses along $Y$. In order to prove the convergence of this method for $p \geq 20 \mathrm{RWs}$, a common procedure in statistics is applied, which is the bootstrapping method. It was introduced in 1977 in [46] to provide estimations of the main statistical properties of the population by considering a reduced sample only. Indeed, given a population $\mathbf{x}$, a bootstrap sample $\mathbf{x}^{*}=\left(x_{1}^{*}, x_{2}^{*}, \ldots, x_{p}^{*}\right)$ is obtained by sampling $n$ times with replacement (e.g.: if $p=7$, one possible bootstrap sample could be $\mathrm{x}^{*}=\left(x_{1}^{*}, x_{2}^{*}, x_{2}^{*}, x_{6}^{*}, x_{5}^{*}, x_{3}^{*}, x_{6}^{*}\right)$. One of the main conditions to obtain a valid bootstrap sample is that it has to be representative of the population. Once $\mathbf{x}^{*}$ is built, $B$ bootstrap replications are run. Being $\theta$ the statistical quantity of the population we are interested in, the bootstrapping method computes the bootstrap sample quantity $\theta^{*}$.

By correctly selecting the number of repetitions $B$ it happens that $\theta^{*} \sim \theta$. [47] suggests to run the method for values of $2000 \leq B \leq 10000$, depending on the complexity of the data. In the case under analysis the bootstrap technique is used to demonstrate the convergence of $M C C M$ response along 
direction $X$ for $p \geq 20$. For this purpose, bootstrapping is run in two different cases as shown in Table 9. Harmonic $h=2$ is considered and the exact value of the response considering the entire family of RWs is such that $\Psi_{X X}(h=2)=3.2 \cdot 10^{-6} g^{2} / H z$. The results are also shown in Figure 17

\begin{tabular}{cccc}
\hline$B$ & $M C C M\left[g^{2} / H z\right]$ & $n$ & Error \\
\hline 5000 & $2.95 \cdot 10^{-6}$ & $20 \mathrm{RWs}$ & $7 \%$ \\
5000 & $2 \cdot 10^{-7}$ & $5 \mathrm{RWs}$ & $93 \%$ \\
\hline
\end{tabular}

Table 9: Bootstrapping results for $\boldsymbol{\Psi}_{\mathbf{X X}}$ at harmonic $h=2$ on SSTL-300. Number of family of RWs $n=30$

where the histograms of the bootstrap results in terms of $M C C M$ response along $X$ are reported. It can be seen that in the case of $p=20$ RWs the pre-

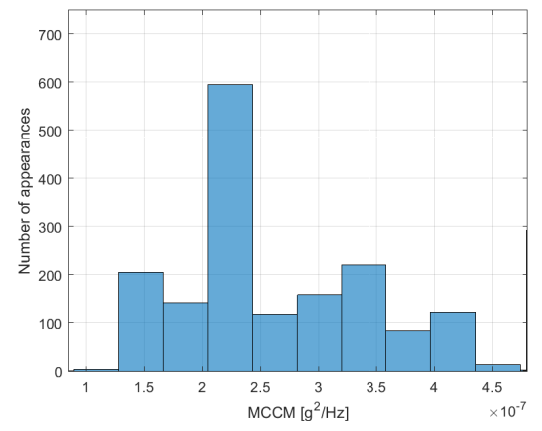

(a) Bootstrapping with $p=5 \mathrm{RWs}$

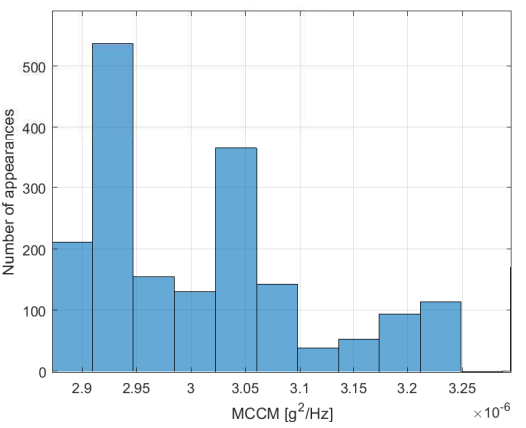

(b) Bootstrapping with $p=20$ RWs

Figure 17: Bootstrapping results for $\boldsymbol{\Psi}_{\mathbf{X X}}$ - Simulation parameters are the same as Table 9. An order of magnitude of difference is present between the 2 cases.

dicted percentage difference in terms of response between the bootstrapped result and the real case is $7 \%$ whereas with a bootstrap samples of $p=5$ RWs it is $93 \%$. Since, as already stated, the bootstrapped sample needs to be representative of the entire population, the results validate this assumption, i.e. $p \geq 20$ RWs is enough to characterise the whole family of RWs.

\section{Conclusions}

In this article a methodology for the characterisation of a family of microvibration sources has been proposed, able to define the generalised dis- 
turbance input matrix $\boldsymbol{\Psi}_{\mathbf{I N}-\max }$ encompassing all the different geometric imperfections of a family of microvibration sources. The main achievements are discussed hereafter. First, $M C C M$ allows for an early microvibration budget estimation: in fact, it provides more accurate, less conservative microvibration predictions based upon typical behaviour of a RW family. Second, with respect to the available approaches for this purpose, mainly $D I A G$ and $S G L$, $M C C M$ significantly decreases the safety factors required in the predictions (by as much as 0.5). Third, the effects of off-diagonal terms in the disturbance input matrix have been studied and their significance in different cases has been examined, assessing the induced variation on the vibration predictions. Beyond that, a quantitative comparison with respect to the simplified and widely accepted case of diagonal matrix has been provided. It has been revealed that in a generic mounting configuration of the wheel on the spacecraft this assumption can lead to significant over/underestimation (a factor $\geq 2$ in some cases). Lastly, it has been shown that this new methodology can characterise the whole family of sources by considering a limited number of them. This last result has practical implications: since the family of sources has to be characterised only partially, there is a significant saving in terms of time. Indeed, each test involves acquisition of the data through dynamometric table and post-processing. Furthermore, the user applying the methodology, only needs a limited amount of data and this can be a primary benefit when data needs to be purchased for a matter of confidentiality and cost.

Despite being the responses generated by $\Psi_{\mathbf{I N}-\mathbf{m a x}}$ still affected by the transfer function matrix $\mathbf{H}$, its effects on a generic structure have shown to be robust and consistent in the different cases studied.

\section{Appendix A. MCCM Convergence Plots and $\Psi_{\mathrm{IN}}$ Matrix Struc- ture}

Here the structure of the $\boldsymbol{\Psi}_{\mathbf{I N}}$ input matrix is reported. In particular the complete matrix $\boldsymbol{\Psi}_{\mathbf{I N - F}}$ and the diagonal one $\boldsymbol{\Psi}_{\mathbf{I N}-\mathbf{D}}$ are reported. In addition, more convergence plots for the $M C C M$ are shown.

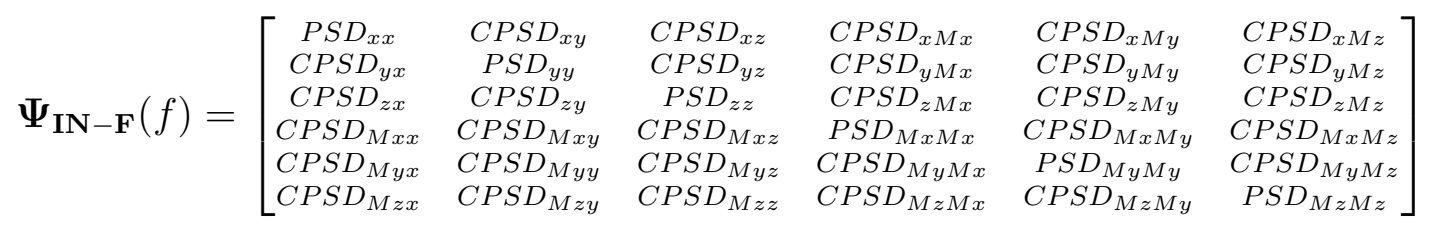




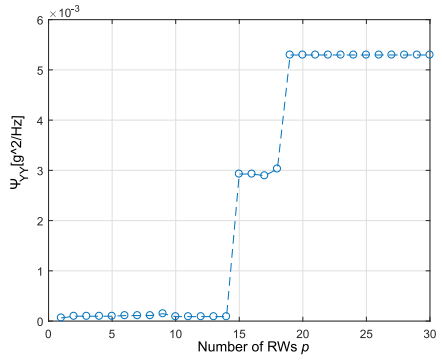

(a) $\boldsymbol{\Psi}_{\mathbf{Y Y}}$ at $h=4.51$

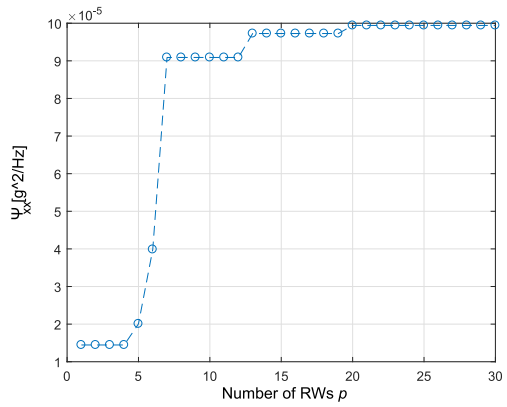

(b) $\boldsymbol{\Psi}_{\mathbf{X X}}$ at $h=10$

Figure A.18: Convergence of the harmonics $h$ for response $\mathbf{\Psi}_{\mathbf{X X}-\text { OUT }}$ and $\mathbf{\Psi}_{\mathbf{Y Y}-\text { OUT }}$ computed on $S S T L-300$ using $M C C M$ with $\mathbf{R}=\mathbf{R}_{\mathbf{3}} * \mathbf{R}_{\mathbf{1}} * \mathbf{R}_{\mathbf{2}}, \theta_{1}=-48^{\circ}, \theta_{2}=121^{\circ}$ and $\theta_{3}=12^{\circ}$.

$\boldsymbol{\Psi}_{\mathbf{I N}-\mathbf{D}}(f)=\operatorname{diag}\left[\boldsymbol{\Psi}_{\mathbf{I N}-\mathbf{F}}(f)\right]$

Note that all the terms contained in the two matrices are a function of the frequency considered.

\section{References}

[1] O. L. de Weck, D. W. Miller, G. E. Mosier, Multidisciplinary analysis of the NEXUS precursor space telescope (December 2002) 294. doi:10.1117/12.460079.

[2] G. S. Aglietti, et al., ECSS-E-HH-32-26A, Spacecraft mechanical loads analysis handbook (2013).

[3] M. Levine, R. Bruno, H. Gutierrez, Interferometry program flight experiment\# 1: Objecties and results.

[4] P. L. Reece, Progress in smart materials and structures, Nova Publishers, 2007.

[5] D. W. Miller, O. L. De Weck, S. A. Uebelhart, R. Grogan, I. Basdogan, Integrated dynamics and controls modeling for the space interferometry mission (SIM), Vol. 4, 2001, pp. 42089-42102. doi:10.1109/AERO.2001.931545. 
[6] J. Betermier, M. Charmeau, N. Jourdon, V. Guillaud, Microvibration tests on satellites-lessons learned from the spot 3 experiment, in: Proceedings of the First International Symposium on Space Microdynamics and Accurate Control, Nice, France, 1992.

[7] T. T. Hyde, K. Q. Ha, J. D. Johnston, J. M. Howard, G. E. Mosier, Integrated modeling activities for the james webb space telescope: optical jitter analysis, in: Optical, Infrared, and Millimeter Space Telescopes, Vol. 5487, International Society for Optics and Photonics, 2004, pp. $588-600$.

[8] K. J. Pendergast, C. J. Schauwecker, Use of a passive reaction wheel jitter isolation system to meet the advanced x-ray astrophysics facility imaging performance requirements, in: Space Telescopes and Instruments V, Vol. 3356, International Society for Optics and Photonics, 1998, pp. 1078-1095.

[9] G. Smet, G. Richardson, S. McLaren, A. Haslehurst, Managing reaction wheel microvibration on a high resolution eo small spacecraft, in: European Space Mechanisms \& Tribology Symposium, ESTEC, Noordwijk, The Netherlands, 2013.

[10] G. Aglietti, R. Langley, E. Rogers, S. Gabriel, Model building and verification for active control of microvibrations with probabilistic assessment of the effects of uncertainties, Proceedings of the Institution of Mechanical Engineers, Part C: Journal of Mechanical Engineering Science 218 (4) (2004) 389-399.

[11] M. Remedia, G. Aglietti, G. Richardson, A stochastic methodology for predictions of the environment created by multiple microvibration sources, Journal of Sound and Vibration 344 (2015) 138-157.

[12] M. S. Kompella, R. J. Bernhard, Measurement of the statistical variation of structural-acoustic characteristics of automotive vehicles, Tech. rep., SAE Technical Paper (1993).

[13] S. Adhikari, M. Friswell, K. Lonkar, A. Sarkar, Experimental case studies for uncertainty quantification in structural dynamics, Probabilistic Engineering Mechanics 24 (4) (2009) 473-492. 
[14] M. Remedia, G. S. Aglietti, G. Richardson, Modelling the effect of electrical harness on microvibration response of structures, Acta Astronautica 109 (2015) 88-102.

[15] S. De Lellis, A. Stabile, G. S. Aglietti, G. Richardson, A preliminary methodology to account for structural dynamics variability of satellites in microvibration analysis, in: 2018 AIAA/ASCE/AHS/ASC Structures, Structural Dynamics, and Materials Conference, 2018, pp. 0454c1.

[16] T. Fang, P. Spoor, S. Ghiaasiaan, M. Perrella, Influence of minor geometric features on stirling pulse tube cryocooler performance, in: IOP Conference Series: Materials Science and Engineering, Vol. 278, IOP Publishing, 2017, p. 012140.

[17] A. Rafsanjani, S. Abbasion, A. Farshidianfar, H. Moeenfard, Nonlinear dynamic modeling of surface defects in rolling element bearing systems, Journal of Sound and Vibration 319 (3-5) (2009) 1150-1174.

[18] N. Jedrich, D. Zimbelman, M. Turczyn, J. Sills, C. Voorhees, B. Clapp, M. Brumfield, Cryo cooler induced micro-vibration disturbances to the hubble space telescope.

[19] H. O. Madsen, S. Krenk, N. C. Lind, Methods of structural safety, Courier Corporation, 2006.

[20] G. E. Musgrave, A. Larsen, T. Sgobba, Safety design for space systems, Butterworth-Heinemann, 2009.

[21] R. J. Balling, J. Sobieszczanski-Sobieski, Optimization of coupled systems-a critical overview of approaches, AIAA journal 34 (1) (1996) $6-17$.

[22] C. Eckert, O. Isaksson, Safety margins and design margins: A differentiation between interconnected concepts, Procedia CIRP 60 (2017) 267-272.

[23] P. Bely, The design and construction of large optical telescopes, Springer Science \& Business Media, 2003. 
[24] S. Lee, E. Skulsky, Mars reconnaissance orbiter design approach for high-resolution surface imaging.

[25] D. Addari, G. S. Aglietti, M. Remedia, Dynamic mass of a reaction wheel including gyroscopic effects: an experimental approach, AIAA Journal 55 (1) (2016) 274-285.

[26] Z. Zhang, G. S. Aglietti, W. Ren, Coupled microvibration analysis of a reaction wheel assembly including gyroscopic effects in its accelerance, Journal of Sound and Vibration 332 (22) (2013) 5748-5765.

[27] D. Addari, G. Aglietti, M. Remedia, Experimental and numerical investigation of coupled microvibration dynamics for satellite reaction wheels, Journal of Sound and Vibration 386 (2017) 225-241.

[28] Z. Zhang, G. S. Aglietti, W. Zhou, Microvibrations induced by a cantilevered wheel assembly with a soft-suspension system, AIAA journal 49 (5) (2011) 1067-1079.

[29] S. Taniwaki, M. Kudo, M. Sato, Y. Ohkami, Analysis of retainer induced disturbances of reaction wheel, Journal of System Design and Dynamics 1 (2) (2007) 307-317.

[30] S. Harsha, Nonlinear dynamic analysis of rolling element bearings due to cage run-out and number of balls, Journal of Sound and Vibration 289 (1-2) (2006) 360-381.

[31] J. Liu, Y. Shao, M. J. Zuo, The effects of the shape of localized defect in ball bearings on the vibration waveform, Proceedings of the Institution of Mechanical Engineers, Part K: Journal of Multi-body Dynamics 227 (3) (2013) 261-274.

[32] S. S. Narayan, P. Nair, A. Ghosal, Dynamic interaction of rotating momentum wheels with spacecraft elements, Journal of Sound and Vibration 315 (4-5) (2008) 970-984.

[33] A. J. Butterfield, S. E. Woodard, Measured spacecraft instrument and structural interactions, Journal of spacecraft and rockets 33 (4) (1996) 556-562. 
[34] K.-C. Liu, P. Maghami, C. Blaurock, Reaction wheel disturbance modeling, jitter analysis, and validation tests for solar dynamics observatory, in: AIAA Guidance, Navigation and Control Conference and Exhibit, 2008, p. 7232 .

[35] I. Basdogan, L. M. Elias, F. Dekens, L. Sievers, Predicting the optical performance of the space interferometry mission using a modeling, testing, and validation methodology, Journal of vibration and acoustics 129 (2) (2007) 148-157.

[36] P. Stoica, R. L. Moses, et al., Spectral analysis of signals, Vol. 1, Pearson Prentice Hall Upper Saddle River, NJ, 2005.

[37] L. M. Elias, F. G. Dekens, I. Basdogan, L. A. Sievers, T. Neville, Methodology for modeling the mechanical interaction between a reaction wheel and a flexible structure, in: Interferometry in Space, Vol. 4852, International Society for Optics and Photonics, 2003, pp. 541-556.

[38] R. Kubo, M. Toda, N. Hashitsume, Statistical physics II: nonequilibrium statistical mechanics, Vol. 31, Springer Science \& Business Media, 2012.

[39] T. A. Harris, M. N. Kotzalas, Essential concepts of bearing technology, CRC press, 2006.

[40] Z. Weiyong, L. Dongxu, L. Qing, L. Kun, Analysis and testing of microvibrations produced by momentum wheel assemblies, Chinese Journal of Aeronautics 25 (4) (2012) 640-649.

[41] R. A. Masterson, D. W. Miller, R. L. Grogan, Development and validation of reaction wheel disturbance models: empirical model, Journal of Sound and Vibration 249 (3) (2002) 575-598.

[42] P. Welch, The use of fast fourier transform for the estimation of power spectra: a method based on time averaging over short, modified periodograms, IEEE Transactions on audio and electroacoustics 15 (2) (1967) $70-73$.

[43] C. M. Harris, A. G. Piersol, Harris' shock and vibration handbook, Vol. 5, McGraw-Hill New York, 2002. 
[44] S. De Lellis, A. Stabile, G. Aglietti, G. Richardson, A methodology for disturbance characterisation of families of microvibration sources, Proceedings of ECSSMET 2018.

[45] Z. Ismail, R. Varatharajoo, A study of reaction wheel configurations for a 3-axis satellite attitude control, Advances in Space Research 45 (6) (2010) 750-759.

[46] B. Efron, Bootstrap methods: another look at the jackknife, in: Breakthroughs in statistics, Springer, 1992, pp. 569-593.

[47] B. Efron, R. J. Tibshirani, An introduction to the bootstrap, CRC press, 1994. 